\title{
Involvement of EupR, a response regulator of the NarL/FixJ family, in the control of the uptake of the compatible solutes ectoines by the halophilic bacterium Chromohalobacter salexigens
}

Javier Rodríguez-Moya ${ }^{1 \dagger}$, Montserrat Argandoña ${ }^{1 \dagger}$, Mercedes Reina-Bueno ${ }^{1}$, Joaquín J Nieto ${ }^{1}$, Fernando Iglesias-Guerra ${ }^{2}$, Mohamed Jebbar ${ }^{3,4}$, Carmen Vargas ${ }^{1 *}$

\begin{abstract}
Background: Osmosensing and associated signal transduction pathways have not yet been described in obligately halophilic bacteria. Chromohalobacter salexigens is a halophilic bacterium with a broad range of salt tolerance. In response to osmotic stress, it synthesizes and accumulates large amounts of the compatible solutes ectoine and hydroxyectoine. In a previous work, we showed that ectoines can be also accumulated upon transport from the external medium, and that they can be used as carbon sources at optimal, but not at low salinity. This was related to an insufficient ectoine(s) transport under these conditions.

Results: A C. salexigens Tn1732-induced mutant (CHR95) showed a delayed growth with glucose at low and optimal salinities, could not grow at high salinity, and was able to use ectoines as carbon sources at low salinity. CHR95 was affected in the transport and/or metabolism of glucose, and showed a deregulated ectoine uptake at any salinity, but it was not affected in ectoine metabolism. Transposon insertion in CHR95 caused deletion of three genes, Csal0865-Csal0867: acs, encoding an acetyl-CoA synthase, $m n t R$, encoding a transcriptional regulator of the DtxR/MntR family, and eupR, encoding a putative two-component response regulator with a LuxR_C-like DNAbinding helix-turn-helix domain. A single $m n t R$ mutant was sensitive to manganese, suggesting that mntR encodes a manganese-dependent transcriptional regulator. Deletion of eupR led to salt-sensitivity and enabled the mutant strain to use ectoines as carbon source at low salinity. Domain analysis included EupR as a member of the NarL/ FixJ family of two component response regulators. Finally, the protein encoded by Csal869, located three genes downstream of eupR was suggested to be the cognate histidine kinase of EupR. This protein was predicted to be a hybrid histidine kinase with one transmembrane and one cytoplasmic sensor domain.
\end{abstract}

Conclusions: This work represents the first example of the involvement of a two-component response regulator in the osmoadaptation of a true halophilic bacterium. Our results pave the way to the elucidation of the signal transduction pathway involved in the control of ectoine transport in C. salexigens.

\section{Background}

Due to the frequent osmolarity changes in their habitat, microorganisms have developed a number of osmoadaptation mechanisms to adapt to these fluctuations [1,2]. In most bacteria, the long-term response to hyperosmotic

\footnotetext{
* Correspondence: cvargas@us.es

+ Contributed equally

'Department of Microbiology and Parasitology, Faculty of Pharmacy,

University of Seville, Seville, Spain

Full list of author information is available at the end of the article
}

conditions involves the intracellular accumulation of large quantities of small, specific organic osmolytes called compatible solutes since they do not interfere with the normal functioning of the cell [3]. It has been demonstrated that compatible solutes have the ability to protect enzymes and whole cells against different stresses such as those caused by salt, heating, freezing and desiccation $[3,4]$. Thus, they are considered as biostabilizers. It is commonly accepted that uptake of exogenous compatible solutes (osmoprotectants) is preferred over their 
synthesis de novo, as it is energetically less costly [5]. On the other hand, hypoosmotic stress leads to opening of mechanosensitive channels, which function as emergence valves leading to rapid efflux of compatible solutes thereby lowering the osmotic driving force for water entry [6]. Besides their role as stress protectants, some compatible solutes can be used as carbon, energy or nitrogen sources. This duality of functions (stress protection and nutrition) requires complex regulatory circuits (most of them not yet elucidated) to adjust the rate of compatible solute biosynthesis, transport and catabolism $[4,7,8]$.

A number of genes and enzymes responsible for synthesis, uptake and efflux of compatible solutes have been identified in diverse bacteria [1,6-10]. However, the mechanisms by which bacteria sense osmotic shifts (osmosensing) and the signal transduction pathways leading to these genes (osmosignaling) have focused on membrane-based osmosensors from moderately halotolerant, but not halophilic, bacteria. These include osmosensory transporters, histidine kinases of two-component transcriptional regulatory systems [9], and mechanosensitive channels of the MscL, MscS and MscK type [6]. Whereas the first and the third group can detect osmotic pressure changes and respond by mediating compatible solute uptake or efflux, respectively, without the assistance of other proteins, membrane-bound histidine kinases detect changes in osmotic pressure and other signals and then respond by directing cognate response regulators to modulate transcription of osmoregulated genes. The best studied osmosensory transporters mediate uptake of potassium, i.e. Trk from Escherichia coli, and betaine, such as ProP from E. coli, OpuA from Lactococcus lactis and BetP from Corynebacterium glutamicum $[9,11]$. On the other hand, the best characterized two-component transcriptional regulatory systems involved in bacterial osmoadaptation are KdpDE and EnvZ/OmpR from E. coli, and MtrAB from C. glutamicum [11-13].

Both sensory histidine protein kinases and response regulators of two-component signal transduction systems are multi-domain proteins. Histidine protein kinases typically consist of a variable $\mathrm{N}$-terminal sensory or "input" domain, which detects environmental stimuli and activates a conserved C-terminal cytoplasmic transmitter domain, comprising an ATP-binding kinase domain and a histidine-containing dimerization domain. On the other hand, most response regulators contain a conserved $\mathrm{N}$-terminal receiver (REC) domain and a variable C-terminal effector or "output" domain. The first one catalyzes the transfer of the phosphoryl group from the cognate histidine protein kinase to one of its own aspartic residues. As a result, the receiver domain undergoes a conformational change capable of promoting activity of the effector domain $[14,16]$.
Two general approaches have been used for classifying bacterial two-component systems. The first one is based on the diversity of input (i.e. cellular location, membrane topology, arrangement of sensory domains) or output (i.e., DNA-binding, RNA-binding, proteinbinding, enzymatic, etc) domain architecture and domain combinations $[14,15,17]$. The second one is based on the phylogeny of transmitter and receiver domains [18]. Interestingly, the results of both classifications agree to a certain extent, as it seems that the majority of signal transduction proteins belong to a relatively small number of major families, which share common ancestry, and gene/domain architecture. Osmosensing and associated signal transduction pathways have not yet been described in obligate halophilic bacteria. Chromohalobacter salexigens [19] is a halophilic gamma proteobacterium that grows optimally at 1.5 $\mathrm{M} \mathrm{NaCl}$ in minimal medium [20]. It requires at least 0.5 $\mathrm{M} \mathrm{NaCl}$ for any growth at all, and can tolerate up to 3 $\mathrm{M} \mathrm{NaCl}$, being considered as a model microorganism to study prokaryotic osmoadaptation [8]. Interestingly, $C$. salexigens lowest salinity for growth is the highest $\mathrm{NaCl}$ concentration that the non halophilic E. coli, traditionally used for osmoregulation studies, can tolerate. $C$. salexigens finely adjusts its cytoplasmic compatible solute pool in order to cope with high salinity and supra-optimal temperatures $[21,22]$. This is achieved by a highly hierarchical accumulation of solutes, dominated by the uptake of external osmoprotectants such as betaine or its precursor choline [23,24], and followed by the synthesis of endogenous solutes, mainly ectoines (ectoine and hydroxyectoine), and minor amounts of glutamate, glutamine, trehalose and glucosylglycerate [8]. Ectoine and hydroxyectoine are essential for osmoprotection and thermoprotection, respectively [22].

C. salexigens can also accumulate ectoines after transport from the external medium, and the ectoine transport rate is maximal at optimal salinity [25]. Within the sequence of the $C$. salexigens genome, we have found orthologs to the TRAP-T-type TeaABC transport system for ectoines of the closely related Halomonas elongata [10]. We have experimental evidence that this system is the main responsible for the uptake of ectoines in C. salexigens (J. Rodriguez-Moya, unpublished data). On the other hand, although glucose is the preferred carbon and energy source, $C$. salexigens can use a wide range of substrates as nutrients, including the compatible solutes betaine, ectoine and hydroxyectoine [25]. Remarkably, neither ectoines nor betaine could support $C$. salexigens growth at low salinity, most probably due to an insufficient uptake of these compatible solutes [25].

Osmoadaptive response through ectoine(s) synthesis in C. salexigens seems to be finely controlled at the transcriptional level, and several general $\left(\sigma^{\mathrm{S}}, \sigma^{32}\right.$, Fur $)$ or 
specific regulators have been described [8,24]. However, the associated sensors remain to be elucidated. In addition, information on osmosensing and signal transduction pathways leading to osmoprotectant uptake in C. salexigens is missing. In this work, we isolated a $C$. salexigens salt-sensitive mutant, strain CHR95, which was nevertheless able to use ectoines as a sole carbon source at low salinities due to a deregulated transport. This mutant was affected in three genes, two of which were transcriptional regulators. Analyses of single mutants affected in these regulators suggested the protein EupR as the response regulator of a two-component system involved in the regulation of ectoine(s) uptake. In addition, we predicted and analyzed its putative sensor histidine kinase. This work establishes the first analysis of the involvement of the response regulator of a two-component system in the osmoadaptive response of halophilic bacteria.

\section{Results}

C. salexigens mutant CHR95 can use ectoines as the sole carbon sources at low salinity

C. salexigens is able to grow in M63 minimal medium with 0.5 to $3 \mathrm{M} \mathrm{NaCl}$. In a search for $C$. salexigens saltsensitive mutants, strain CHR95 was isolated after Tn1732 transponson mutagenesis, as being able to grow at $0.5 \mathrm{M}$ but not at $2.7 \mathrm{M} \mathrm{NaCl}$ on $\mathrm{M} 63$ plates (see Methods). To further characterize its salinity range, C. salexigens wild type and CHR95 strains were grown in M63 minimal medium with $20 \mathrm{mM}$ glucose as the sole carbon source, at salinities ranging from 0.6 to 2.5 $\mathrm{M} \mathrm{NaCl}$. As shown in Figure 1 , at $0.6 \mathrm{M} \mathrm{NaCl}$ the growth curve of strain CHR95 showed a $20 \mathrm{~h}$ lag phase, followed by a sharp exponential phase to reach the same $\mathrm{OD}_{600}$ as the wild type strain after ca. $30 \mathrm{~h}$ of growth (see Table 1 for growth rates). At $0.75 \mathrm{M}$ and $1.5 \mathrm{M}$ $\mathrm{NaCl}$, growth of the mutant followed a similar pattern,

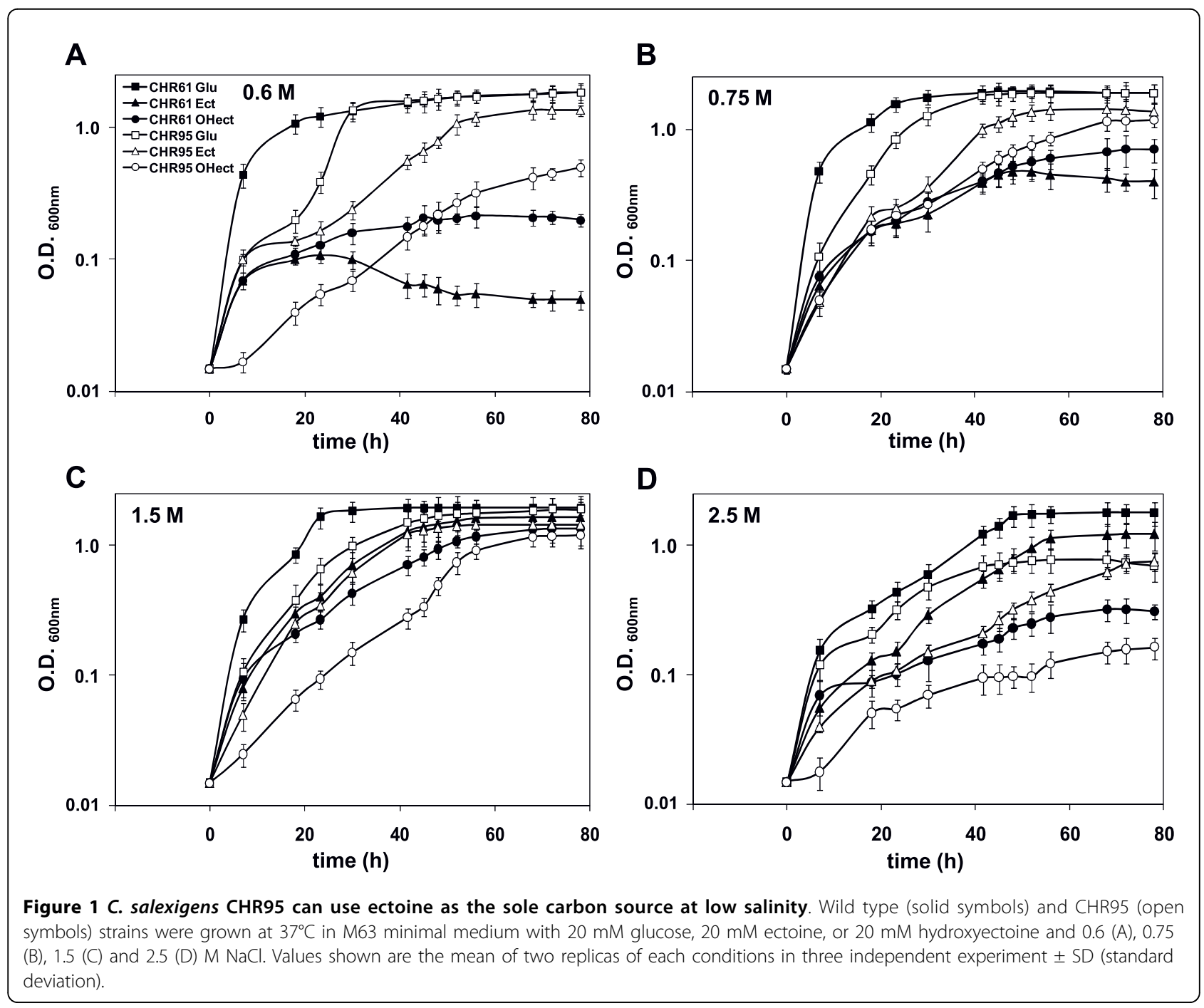


i.e., an extended lag phase, followed by a less pronounced exponential phase than that of the wild type strain, to eventually reach the wild type growth curve at the stationary phase of growth. At $2.5 \mathrm{M} \mathrm{NaCl}$ the strain CHR95 showed a salt-sensitive phenotype, as its growth curve did not reach an $\mathrm{OD}_{600}$ above 0.6 units (Figure 1 and Table 1).

We also compared the ability of the $C$. salexigens wild type strain and mutant CHR95 to use ectoine and hydroxyectoine as the sole carbon sources at different salinities. As shown in Figure 1 and Table 1, in all growth experiments ectoine was better carbon source than hydroxyectoine. Ectoine and hydroxyectoine did not support the growth of the wild type strain at low salinity $(0.6 \mathrm{M} \mathrm{NaCl})$, and growth was severely impaired at $0.75 \mathrm{M} \mathrm{NaCl})$. They were used as carbon sources at optimal $(1.5 \mathrm{M} \mathrm{NaCl})$ and high $(2.5 \mathrm{M} \mathrm{NaCl})$ salinity (in

\section{Table 1 Growth rates of $C$. salexigens wild type strain (CHR61) and mutant CHR95 on glucose and ectoines at different salinities}

\begin{tabular}{ll}
\hline Strain and carbon source & Growth rate $\left.\mathbf{~} \mathbf{~}^{-1}\right)$ \\
\hline CHR61 glucose & \\
$0.6 \mathrm{M}$ & 0.043 \\
$0.75 \mathrm{M}$ & 0.066 \\
$1.5 \mathrm{M}$ & 0.100 \\
$2.5 \mathrm{M}$ & 0.061 \\
CHR61 ectoine & \\
$0.6 \mathrm{M}$ & 0 \\
$0.75 \mathrm{M}$ & 0.013 \\
$1.5 \mathrm{M}$ & 0.045 \\
$2.5 \mathrm{M}$ & 0.032 \\
CHR61 hydroxyectoine & \\
$0.6 \mathrm{M}$ & 0 \\
$0.75 \mathrm{M}$ & 0.012 \\
$1.5 \mathrm{M}$ & 0.030 \\
$2.5 \mathrm{M}$ & 0.007 \\
CHR95 glucose & \\
$0.6 \mathrm{M}$ & 0.090 \\
$0.75 \mathrm{M}$ & 0.055 \\
$1.5 \mathrm{M}$ & 0.044 \\
$2.5 \mathrm{M}$ & 0.007 \\
CHR95 ectoine & \\
$0.6 \mathrm{M}$ & 0.038 \\
$0.75 \mathrm{M}$ & 0.045 \\
$1.5 \mathrm{M}$ & 0.046 \\
$2.5 \mathrm{M}$ & 0.020 \\
CHR95 hydroxyectoine & \\
$0.6 \mathrm{M}$ & 0.010 \\
$0.75 \mathrm{M}$ & 0.023 \\
$1.5 \mathrm{M}$ & 0.045 \\
\hline $.5 \mathrm{M}$ & 0 \\
\hline
\end{tabular}

this latter case, only ectoine and after a prolonged lag phase). Remarkably, mutant CHR95 was able to use ectoine and hydroxyectoine as the sole carbon and energy source at low salinities (0.6-0.75 $\mathrm{M} \mathrm{NaCl}$ ), although growth with hydroxyectoine was initiated after a long lag phase (Figure 1 and Table 1). Other compatible solutes like glycine betaine were not metabolized under low salinity conditions (not shown). At $1.5 \mathrm{M}$ $\mathrm{NaCl}$ with ectoine or hydroxyectoine, growth of the mutant was delayed, if compared to the wild type strain, whereas at $2.5 \mathrm{M} \mathrm{NaCl}$ ectoine or hydroxyectoine did weakly support or not, respectively, CHR95 growth (Figure 1 and Table 1).

Given that strain CHR95 showed a delayed growth with glucose at any salinity tested, we used natural abundance ${ }^{13} \mathrm{C}$-NMR to determine the total pool of compatible solutes accumulated by cells grown in M63 with $2.5 \mathrm{M} \mathrm{NaCl}$. The ${ }^{13} \mathrm{C}-\mathrm{NMR}$ spectrum of the mutant contained four sets of resonances that were assigned to ectoine, hydroxyectoine, glutamate and glutamine (not shown). This observation suggested that CHR95 was not affected in the genes encoding the synthesis of compatible solutes.

\section{Mutant CHR95 is affected in the transport and metabolism of glucose}

Since, if compared to the wild type strain, strain CHR95 showed delayed growth with glucose at low and optimal salinity, we analyzed the metabolism of glucose in both strains. For this purpose, cells were cultivated in M63 with $1.5 \mathrm{M} \mathrm{NaCl}$, and the fate of radioactive glucose was determined at different time intervals as described in Methods (Figure 2). First, the total radioactivity remaining in supernatant $(S)$ was determined and considered as an indirect measure of glucose transport. As evidenced by the sharp decrease in the radioactivity remaining in the supernatant, the wild type strain incorporated about $95 \%$ of the glucose from 20 (early exponential phase) to 38 hours of incubation. In contrast, glucose uptake by the mutant was slower, with 10-fold higher radioactivity levels in its supernatant than those of the wild type after 38 hours of incubation (Figure 2a). Second, we determined, for the wild type and CHR95 strains, the radioactivity present in the ethanol insoluble fraction (EIF), containing cell envelopes and intracellular macromolecules (lipids, proteins), and the ethanol soluble fraction (ESF), containing small cytoplasmic organic solutes (including ectoines, amino acids, and others). From the same time interval comprised between 20 and 38 hours of incubation, the radioactivity present in the EIF and the ESF of strain CHR95 was 1.5 to 1.8-fold lower (Figure 2b), and 1.3-fold lower (Figure 2c), respectively, than those of the wild type strain. These results, taken together, suggest that the slow 


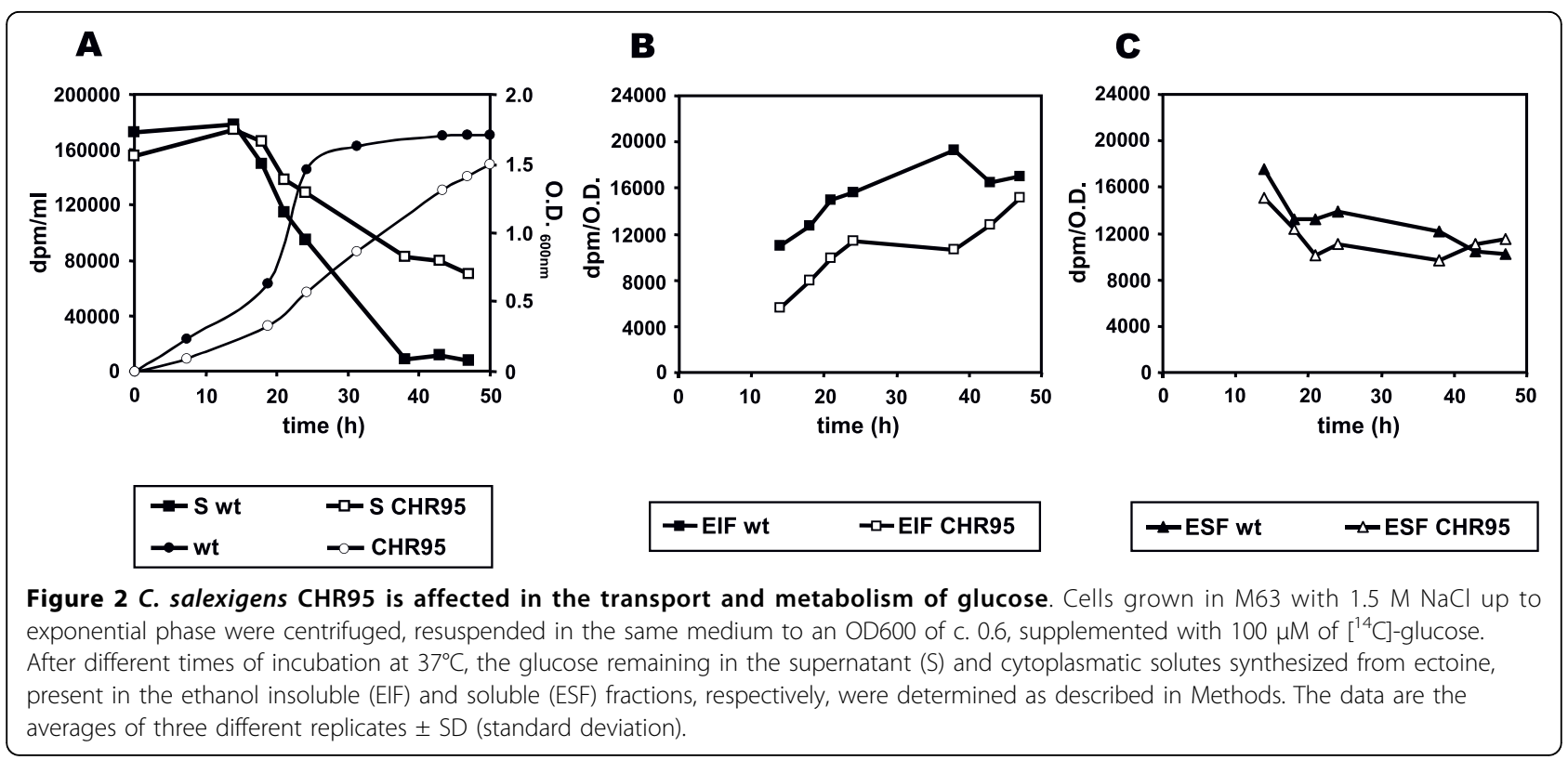

growth of strain CHR95 with glucose might be due, at least in part, to a decreased glucose transport and metabolism.

Mutant CHR95 possesses a deregulated ectoine uptake As mutant CHR95, but not the wild type strain, could use ectoines as nutrients at low salinities, we investigated the transport and metabolism of ectoine in both strains in response to increasing osmolarity. As

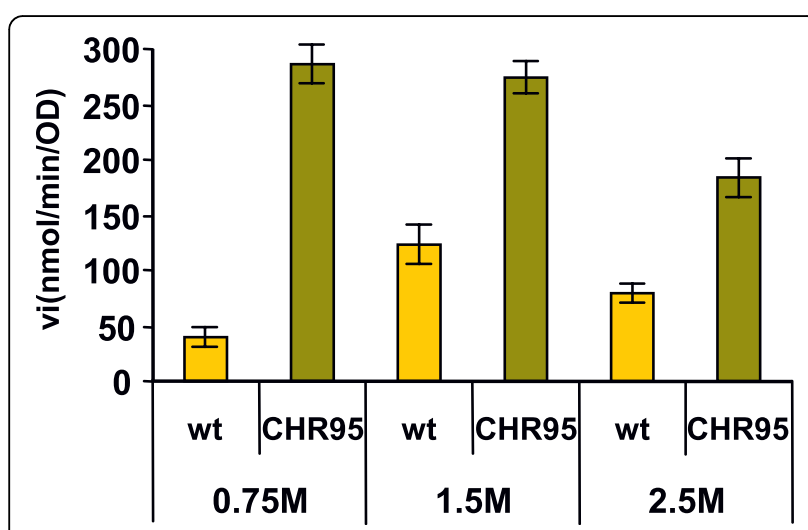

Figure 3 C. salexigens CHR95 shows a deregulated ectoine uptake. The wild-type strain and the mutant CHR95 ( $\triangle$ acseupRmntR::Tn1732) were grown in glucose M63 minimal medium containing the indicated concentration of $\mathrm{NaCl}$. The measurement of $40\left[\left[^{14} \mathrm{C}\right]\right.$-ectoine uptake rates (vi, expressed as $\mathrm{nmol}$ $\mathrm{min}^{-1} \mathrm{OD}^{-1}$ units) was performed as described in Methods. Experiments were repeated twice, and the data correspond to mean values. previously reported by Vargas et al [25], the wild type strain showed its maximal ectoine transport rate at the optimal salinity for growth $(1.5 \mathrm{M} \mathrm{NaCl})$, which was 3- and 1.5-fold higher than those observed at 0.75 and $2.5 \mathrm{M} \mathrm{NaCl}$, respectively (Figure 3). Notably, the ectoine transport rates of strain CHR95 were 8-, 2.3-, and 2.5 -fold higher at $0.75,1.5$, and $2.5 \mathrm{M} \mathrm{NaCl}$, respectively, than those of the wild type grown at the same salt concentrations (Figure 3).

To test if the metabolism of ectoine was affected in $\mathrm{CHR} 95$, the fate of radioactive ectoine was analysed in the presence or absence of $20 \mathrm{mM}$ glucose as described in Methods, and compared to that of the wild type strain. According to previous studies [25], $\mathrm{CO}_{2}$ production due to ectoine catabolism in the wild type strain was lower (40-fold) in the presence of glucose, suggesting that ectoine utilization is partially repressed by glucose. No significant differences were found between $\mathrm{CO}_{2}$ production from ectoine by CHR95 and the wild type strain, neither with nor without glucose addition (Figure 4a). In both strains, most of the carbon backbone of ectoine (ca. $70 \%$ of the total radioactivity added) was found in the ethanol soluble fraction (ESF), whereas only about $3.82 \%$ of the total radioactivity added was found in the ethanol insoluble fraction (EIF). No significative differences were found in the radioactivity present in the ESF and EIF fractions of the wild type and mutant strain. Glucose did not influence the biosynthesis of molecules from ectoine in any of these fractions (Figure $4 \mathrm{~b}$ ). These results suggested that whereas ectoine transport is deregulated in mutant CHR95 at any salinity, ectoine metabolism is not affected in this strain. 


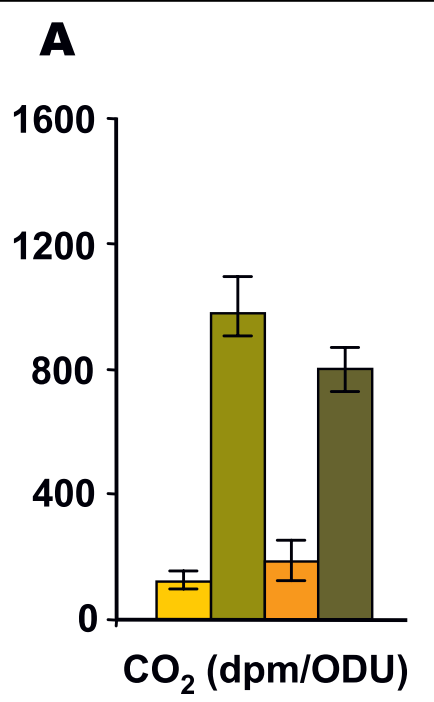

B

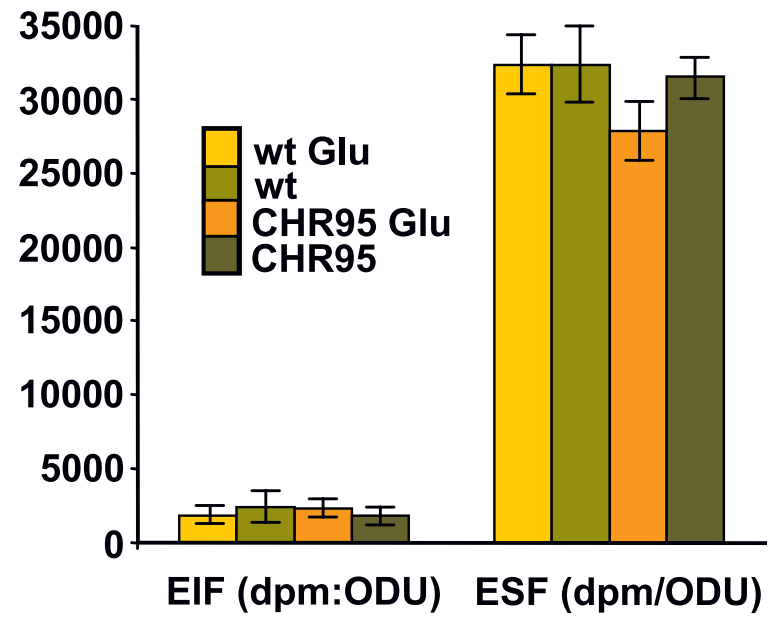

Figure 4 C. salexigens CHR95 is not affected in the metabolism of ectoine. Cells grown in $\mathrm{M} 63$ with $1.5 \mathrm{M} \mathrm{NaCl}$ up to exponential phase were centrifuged, resuspended in the same medium to an $\mathrm{OD}_{600}$ of ca. 0.6, supplemented with $87 \mu \mathrm{M}$ of [ $\left.{ }^{14} \mathrm{C}\right]$-ectoine and incubated with and without $20 \mathrm{mM}$ of glucose. After $2 \mathrm{~h}$ incubation at $37^{\circ} \mathrm{C}, \mathrm{CO}_{2}$ production from ectoine (A) and macromolecules (ElF, B) and cytoplasmatic solutes (ESF, C) synthesized from ectoine, present in the ethanol insoluble and soluble fractions, respectively, were determined as described in Methods. The data are the averages of three different replicates \pm SD (standard deviation).

Transposon insertion in mutant CHR95 caused deletion of genes for the acetyl-CoA synthase and two transcriptional regulators

The salt sensitivity of strain CHR95, together with its altered glucose metabolism and its capacity to use ectoines as carbon sources at low salinity, prompted us to analyze the gene(s) that was(were) affected by the Tn1732 insertion in this mutant. For this purpose, the DNA region flanking the insertion was cloned in plasmid pRR1, which was shown to carry Tn1732 (6.7-kb) plus about $14 \mathrm{~kb}$ of adjacent DNA. To exactly localize the gene(s) disrupted by the transposon, the DNA region flanking the insertion was sequenced by using Tn1732 internal primers. As shown in Figure 5, three genes were deleted by the Tn1732 insertion, named as Csal0865, Csal0866, and Csal0867 within the C. salexigens genome sequence. Csal0865and Csal0866 were located in the forward strand and separated by a 260-bp intergenic region, whereas Csal0867 was located in the complementary strand. The product of Csal0865 (hereafter Acs) was annotated as an acetyl CoA synthase, which activates acetate to acetyl-CoA. In an iterative PSIBLAST search, it showed ca $70 \%$ of amino acid identity to proteins annotated as acetyl CoA synthases from Rhodopseudomonas palustris and Vibrio cholerae. Genes Csal0866 and Csal0867 were predicted to encode putative transcriptional regulators. Thus, the Csal0866 product (hereafter EupR) was annotated as a "two-component LuxR family transcriptional regulator". An iterative PSI-BLAST search revealed a high identity (ca. 65-70\%) to proteins annotated as response regulators of gamma (i.e.
Vibrio, Pseudomonas, Shewanella, Marinobacter, Aeromonas) and alpha (ie. Bradyrhizobium, Labrenzia) proteobacteria. On the other hand, the protein encoded by Csal0867 (hereafter MntR) showed a high identity to manganesedependent transcriptional regulators of the DtxR/MntR family such as MntR of E. coli. Moreover, it showed the characteristic domains of these metalloregulators, i.e., an $\mathrm{N}$-terminal helix-turn-helix domain and a C-terminal metal binding and dimerisation domain. $m n t R$ was preceded by two genes encoding a putative sensor histidine kinase (Csal869) and a putative manganese transporter (MntH), respectively. An in silico analysis of promoter and transcriptional terminator regions showed two putative $\sigma^{70}$-dependent promoters (one upstream of eupR and another one upstream of the sensor histidine kinaseencoding gene), and a putative Rho-independent transcriptional terminator downstream of eupR.

\section{The $C$. salexigens $M n t R$ regulator is involved in the} control of manganese uptake

In other bacteria, such as Bacillus subtilis, MntR is a manganese-dependent metalloprotein involved in the regulation of manganese uptake. $m n t R$ mutants are manganesesensitive since MntR represses genes encoding $\mathrm{Mn}$ (II) transporters. Thus, in the absence of MntR, manganese uptake is deregulated and therefore manganese is toxic to the cells [26]. Since the gene Csal0867 (encoding a putative MntR/DtxR-like global transcriptional regulator) was deleted by the Tn1732 insertion in strain CHR95, we generated a mntR strain (CHR161), in which the gene 


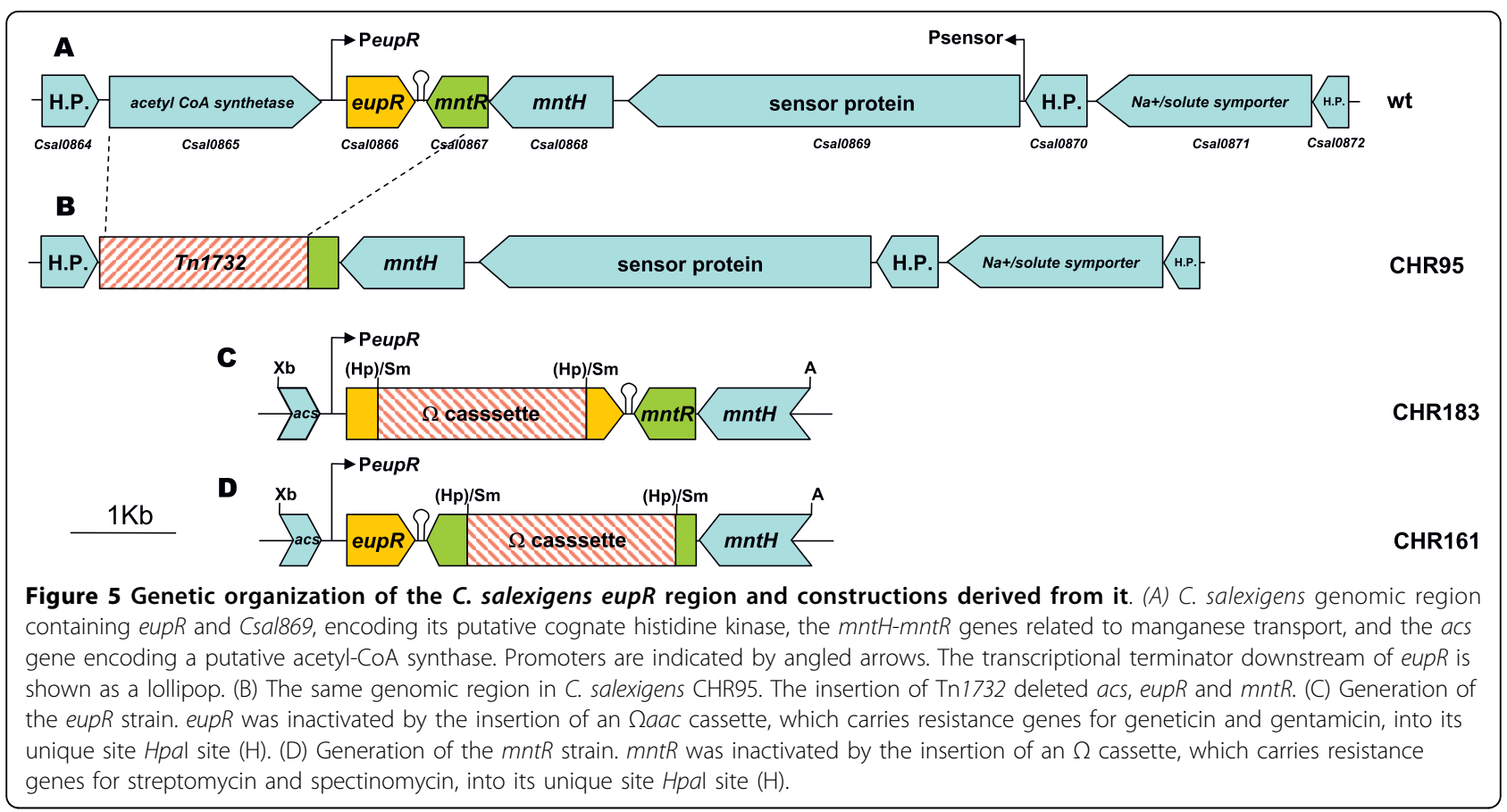

encoding this transcriptional regulator was interrupted by an omega cassette (Figure 5), and investigated its sensitivity to manganese. The wild type, $m n t R$, and CHR95 strains were plated on modified SW-2 plates with different $\mathrm{MnCl}_{2}$ concentrations ranging from 0.5 to $2.5 \mathrm{mM}$. As expected, mutants CHR95 and CHR161 (mntR) did not grow with any $\mathrm{MnCl}_{2}$ concentration (Figure 6). This finding, together with the in silico analysis of the motifs in the protein encoded by Csal0867, suggested that the $m n t R$ gene might encode a manganese-dependent transcriptional regulator.

\section{Deletion of the eupR gene in the CHR95 mutant is responsible for deregulation of ectoine uptake}

The results presented so far suggested that at least one of the genes affected by the Tn1732 transposon insertion in $C$. salexigens CHR95 could be involved in the regulation of ectoine uptake. Besides the gene encoding the MntR regulator, the gene Csal0866 (eupR), encoding a response regulator of a two-component system, was deleted by the Tn1732 insertion in CHR95 (Figure 5). Thus, we generated a mutant affected in this transcriptional regulator (CHR183) and tested its capacity to use ectoines as carbon source at low salinity, as a measure of enhanced transport at this salinity. The wild type and CHR161 (mntR) strains were also included in the assay for comparative purposes. Strains were grown in M63 medium with glucose, ectoine or hydroxyectoine as the sole carbon sources, at salinities ranging from 0.6 to 2.5 $\mathrm{M} \mathrm{NaCl}$. No significant differences were found between the growth of the mntR mutant and the wild type strain with any carbon source at any salinity tested (Figure 7 and Table 2). In contrast, mutant CHR183 (Csal0866) reproduced the phenotype of strain CHR95 and was able to use ectoine and, to a lower extent, hydroxyectoine as the sole carbon and energy sources at low salinity (Figure 7 and Table 2). Like strain CHR95, and if compared to the wild type, growth of CHR183

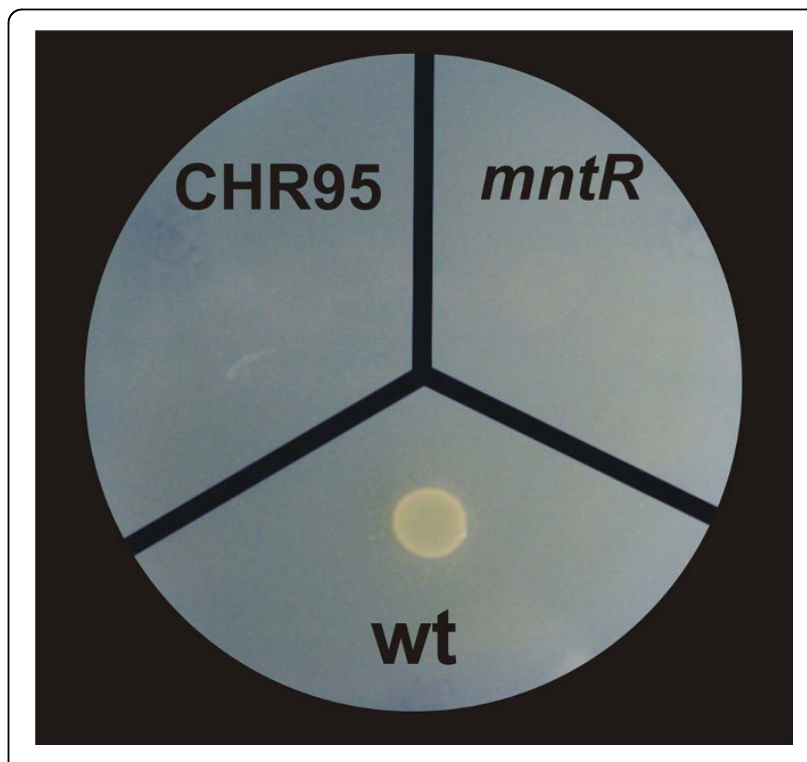

Figure 6 C. salexigens MntR is involved in the control of manganese uptake. $100 \mu \mathrm{L}$ of overnight cultures of the wild type, CHR95 (DacseupRmntR::Tn1732) and CHR 161 (mntR:: $\Omega$ ) were placed on SW2 plates with $0.5 \mathrm{mM} \mathrm{MnCl}_{2}$ and growth was observed after incubation at $37^{\circ} \mathrm{C}$ for $48 \mathrm{~h}$. 

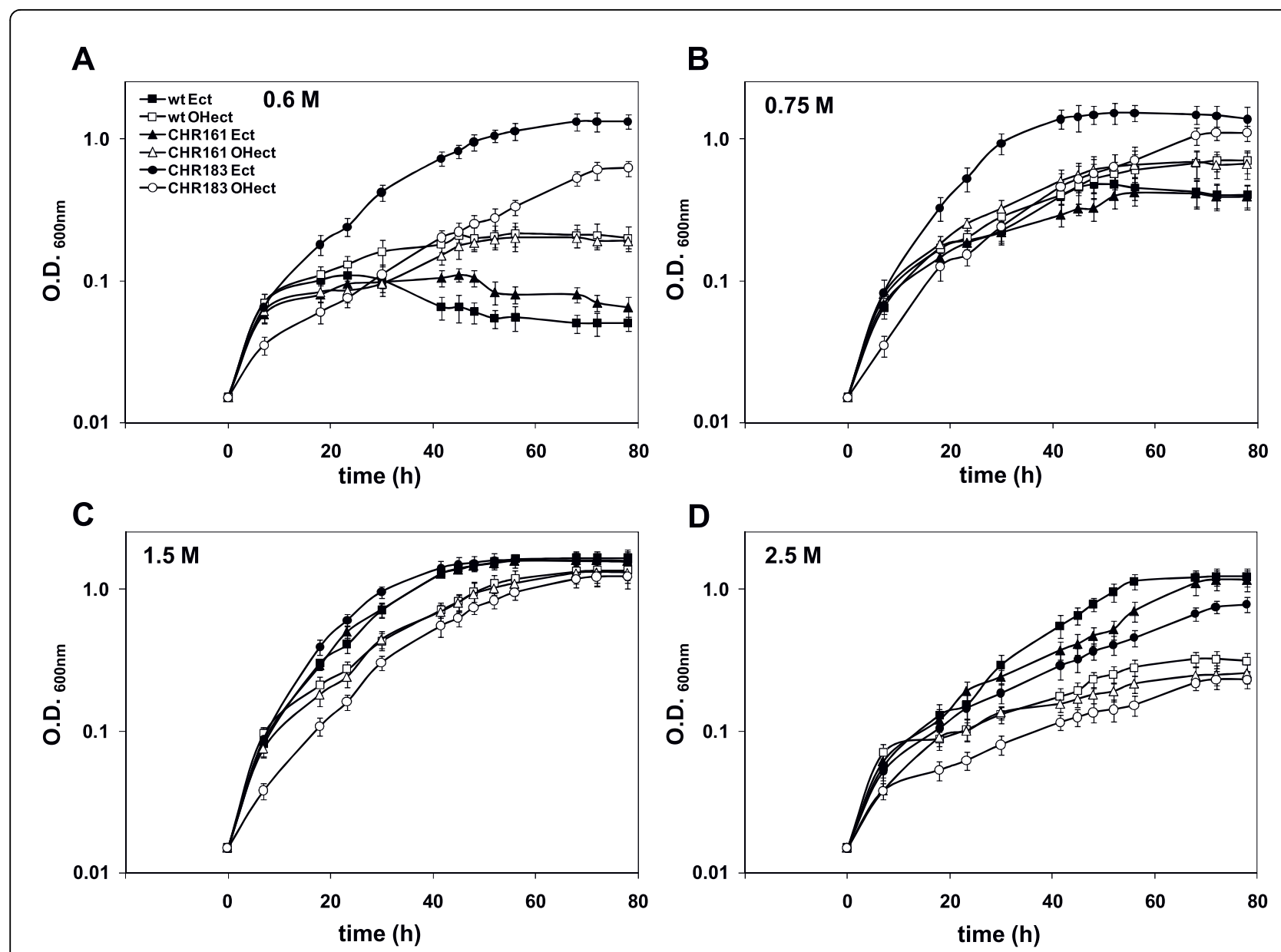

Figure 7 C. salexigens EupR is involved in the control of ectoine uptake. Wild type strain (squares), CHR161 mutant (mntR:: $\Omega$ ) (triangles) and CHR183 mutant (eupR:: $\Omega$ aac) (circles) were grown at $37^{\circ} \mathrm{C}$ in $\mathrm{M} 63$ medium with $20 \mathrm{mM}$ ectoine (black markers) or 20 mM hydroxyectoine (white markers) and 0.6 (A), 0.75 (B) or 1.5 (C) $\mathrm{M} \mathrm{NaCl}$. Values shown are the mean of two replicas of each condition in three independent experiment \pm SD (standard deviation).

(Csal0866) with glucose was delayed from 0.6 to $1.5 \mathrm{M}$ $\mathrm{NaCl}$, and severely impaired at $2.5 \mathrm{M} \mathrm{NaCl}$ (data not shown). The above findings suggest that deletion of gene Csal0866 enables the strain to use ectoines as carbon source at low salinity, as a consequence of ectoine transport deregulation at this salinity. Therefore, the product of Csal0866 was named EupR (after Ectoine uptake Regulator).

\section{EupR is a response regulator of the NarL/FixJ family of proteins}

To further characterize EupR, we analyzed in detail its domain composition and its phylogenetic relationship with other proteins showing the same DNA-binding domain. First, both NCBI/CDD and UniProt entries for this protein included an $\mathrm{N}$-terminal signal receiver domain (REC) and a LuxR_C-like DNA-binding helixturn-helix (HTH) domain. All first 50 hits of the list retrieved after iterative PSI-BLAST, inspected with the CDD domain viewer [27], also showed the same domain composition. Second, we searched Csal866 annotation in the specialized Signaling Census database (see Methods), which includes total counts of signal transduction proteins in completely sequenced genomes [28,29]. In this database, Csal866 was included as a response regulator of the NarL family. As a matter of fact, published classifications of response regulators based on effector domains refer to NarL-like or NarL/FixJ-like proteins to include response regulators with a REC and a HTH DNA-binding domain, as the first structurally characterized HTH domain was from E. coli NarL $[14,17]$. The DNA-binding C-terminal HTH domain of NarL-like proteins was further proposed as a member of the superfamily of the LuxR_C-like DNA-binding HTH domains [30]. Thus, we made a phylogenetic analysis of EupR and related proteins, all containing the common 


\begin{tabular}{|c|c|}
\hline Strain and carbon source & Growth rate $\left(h^{-1}\right)$ \\
\hline \multicolumn{2}{|l|}{ CHR161 ectoine } \\
\hline $0.6 \mathrm{M}$ & 0 \\
\hline $0.75 \mathrm{M}$ & 0.011 \\
\hline $1.5 \mathrm{M}$ & 0.041 \\
\hline $2.5 \mathrm{M}$ & 0.029 \\
\hline \multicolumn{2}{|l|}{ CHR161 hydroxyectoine } \\
\hline $0.6 \mathrm{M}$ & 0 \\
\hline $0.75 \mathrm{M}$ & 0.012 \\
\hline $1.5 \mathrm{M}$ & 0.024 \\
\hline $2.5 \mathrm{M}$ & 0 \\
\hline \multicolumn{2}{|l|}{ CHR183 ectoine } \\
\hline $0.6 \mathrm{M}$ & 0.033 \\
\hline $0.75 \mathrm{M}$ & 0.044 \\
\hline $1.5 \mathrm{M}$ & 0.040 \\
\hline $2.5 \mathrm{M}$ & 0.016 \\
\hline \multicolumn{2}{|l|}{ CHR183 hydroxyectoine } \\
\hline $0.6 \mathrm{M}$ & 0.015 \\
\hline $0.75 \mathrm{M}$ & 0.021 \\
\hline $1.5 \mathrm{M}$ & 0.023 \\
\hline $2.5 \mathrm{M}$ & 0 \\
\hline
\end{tabular}

LuxR_C-like domain. These included well characterized response regulators as well as other homologous but uncharacterized proteins revealed by PSI-BLAST searches, two EupR paralogs present in the C. salexigens genome (also classified in the Signaling Census database as response regulators of the NarL family), and "true" LuxR transcriptional regulators related to quorum sensing. All these proteins were aligned by using ClustalW and the phylogenetic tree was constructed using the Neighbor-joining algorithm of the MEGA 4 software. As shown in Figure 8, the vast majority of the proteins were grouped into two subtrees or families. The first subtree comprised two-component response regulators of the NarL/FixJ family, including well characterized proteins such as the $S$. meliloti FixJ regulator (controlling nitrogen fixation genes [31]), the E. coli UhpA regulator (controlling the UhpT sugar phosphate transport system [32]), and the E. coli NarL protein that controls nitrate- and nitrite-regulated gene expression [33]. All proteins in the first family showed the $\mathrm{N}$-terminal signal receiver phosphoacceptor domain (REC) and the LuxR_C-like domain. Within this family, C. salexigens EupR formed a separated branch with other three proteins of unknown function from Pseudomonas putida, Aeromonas salmonicida and Vibrio harveyi. The EupR paralog Csal_2132 (YP 574182) was closely related to the BvgA virulence factors transcription regulator from Bordetella pertussis (unpublished), whereas the EupR paralog Csal_3030 (YP 575073) was related to the S. meliloti FixJ regulator [31]. The second family included transcriptional regulators that were not response regulators of two components systems, but proteins related to quorum sensing mechanisms. These proteins shared the LuxR_C-like DNA binding domain but showed an $\mathrm{N}$-terminal autoinducer binding domain typical of quorum sensing regulators. Although all these regulators are involved in quorum sensing mediated responses, they control a wide variety of cellular functions, from elastase expression in the case of $P$. aeruginosa LasR [34] to antibiotic production in the case of P. carotovorum CarR [35]. The remaining proteins formed separated and independent branches and only showed the LuxR_C-like DNA binding domain. They were involved in different functions like sporulation control as GerE from B. subtilis [36] or biofilm formation as PsoR from P. putida [37]. All these data indicate that EupR is a response regulator of two-component regulatory systems of the NarL/FixJ family of proteins.

\section{Identification and analysis of the sensor histidine kinase putatively associated to EupR}

The classical two-component regulatory systems require a response regulator protein and a sensor protein, usually a membrane-bound sensor histidine protein kinase [16]. To identify the cognate histidine kinase of EupR, we used the the online application STRING 8.2 (http://string.embl.de/; [38]), a database and web resource dedicated to predict protein-protein interactions including both physical and functional interactions. STRING uses prediction algorithms based on data of neighborhood, gene fusion and co-occurrence across genomes, among others. A total of 21 histidine protein kinases and 29 response regulators are included in the genome of C. salexigens (http://www.ncbi.nlm.nih.gov/ Complete_Genomes/SignalCensus.html) but only the protein encoded by Csal869, located three genes downstream EupR (see Figure 5), was connected with EupR by STRING with a high confidence score $(0.772$, composed of a neighborhood score of 0.193 and a co-occurrence across genomes score of 0.736). Predictions based on STRING algorithms do not have the specificity of experimental data, but have enough statistical robustness as to be considered reliable [38].

To make a deeper functional in silico analysis of this signal transduction protein, we first compared it against several domain databases (see Methods). As Figure 8b shows, we found five distinct domains in the protein: two N-terminal "input" or sensor domains (SSF and PAS-PAC), a transmitter C-terminal region with a Hiscontaining phosphoaceptor HiskA domain and an ATPbinding HATPase domain, and a C-terminal signal receiver domain (REC). The key residues (active site) 


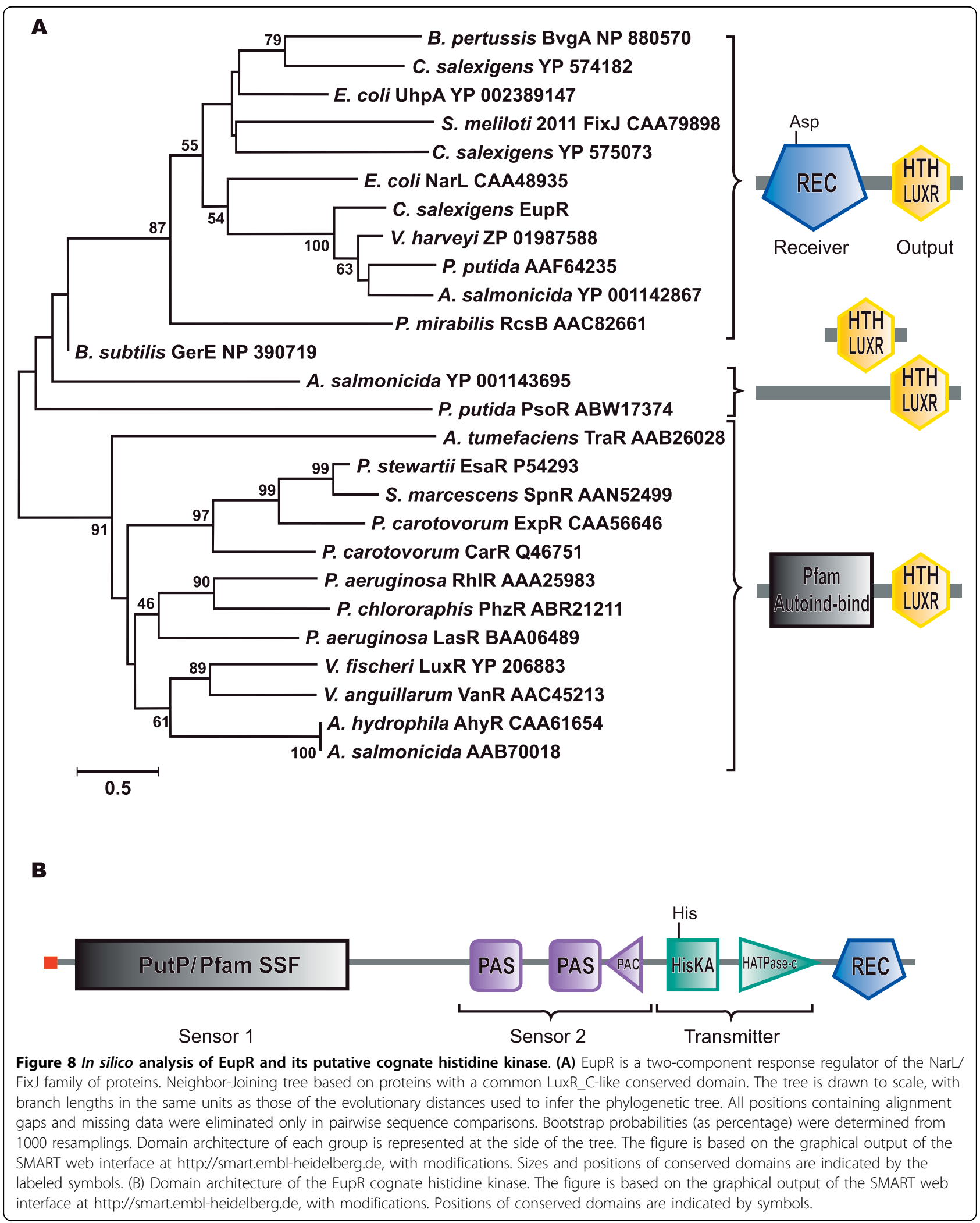


were conserved in HiskA, HATPase and REC domains. Thus, this protein may be classified as a multi-sensor hybrid histidine kinase. In fact, it was included as such in the Signaling Census database [28,29]. Although sensory domains of histidine kinases are extremely diverse, members of the same family domain typically recognize the same (or very close) substrates [39]. Therefore, we anticipated that the analysis of the two sensory domains in our histidine kinase could help us to predict its putative function. The first one showed homology to transmembrane sensory domains like PutP $(\mathrm{Na}+$ /proline symporter-like, in COG database) and SSF (sodium/ solute symporter family, in Pfam database). It was preceded by a signal peptide and predicted to form twelve transmembrane helices. The second one, predicted to be cytoplasmatic, showed two PAS subdomains followed by a C-terminal PAC subdomain. In summary, the putative cognate histidine kinase of EupR was predicted to be a hybrid histidine kinase with both transmembrane and cytoplasmic sensor domains, suggesting that it could sense both external and internal conditions, and integrate them. Moreover, our in silico analysis supports the hypothesis that it may be the sensor partner of EupR.

\section{Discussion}

In this work, we have characterized the Tn1732-induced salt-sensitive mutant CHR95 of C. salexigens, which showed a multiple affected phenotype: (i) inability to grow with glucose at high salinity, but not affection in the synthesis of compatible solutes, (ii) a slow growth with glucose at low and optimal salinity, (iii) a reduced uptake and metabolism of glucose, (iv) a deregulated ectoine uptake at any salinity, and specially at low salinity, but unaffected ectoine metabolism, and (v) sensitivity to manganese. This pleiotropic phenotype was due to deletion of three genes by the insertion of $\operatorname{Tn} 1732$, acs, encoding a putative acetyl-CoA synthase, $m n t R$, encoding a manganese-dependent transcriptional regulator of the DtxR/MntR family, and eupR, encoding a twocomponent response regulator of the NarL/FixJ family of transcriptional regulators. Transposon $\operatorname{Tn} 1732$ is a derivative of $\operatorname{Tn} 1721$, which in turn is a member of the $\operatorname{Tn} 21$ subgroup of the $\operatorname{Tn} 3$ family [40]. It has been widely used for generalized insertion mutagenesis in strains of Halomonas and Chromohalobacter, yielding single mutants [41]. However, as any Tn1721-derivative, it may cause deletions and inversions [42]. Thus, deletion of the region comprising acs-eupR-mntR upon Tn1732 insertion in CHR95 is not surprising. In fact, in the same mutagenesis experiment in which $\mathrm{CHR} 95$ was isolated, we also isolated the salt-sensitive mutant CHR62, showing a deletion of the ectABC genes [21,22].

Whereas the sensitivity of strain CHR95 to manganese was correlated with the absence of $m n t R$, its inability to grow with glucose at high salt, and the reduced transport and metabolism of glucose at low and optimal salinity (leading to a slow growth with this carbon source) may be related to deletion of the acs and/or eupR genes. The physiological role of the Acs enzyme is to activate acetate to acetyl-coenzyme A (Ac-CoA), providing the cell with the two-carbon metabolite used in many anabolic and energy generation processes [43]. Besides Acs (YP_572921), C. salexigens genome encodes at least one protein (YP_573871) showing a PRK03584 domain of Ac-CoA synthases, and also two more proteins with putative acyl CoA synthase domains (YP_573520 and YP_574569). One or more of these proteins might compensate the lack of Acs in CHR95. In addition, it has been reported that prokaryotic cells have evolved different pathways to obtain Ac-CoA, some of them independent of the acs gene [43]. Therefore, with the present data we cannot conclude that deletion of the acs gene influenced the ability of strain CHR95 to grow with glucose as the sole carbon source. The role of the response regulator EupR in such a phenotype seems to be more clearly established, as a single eupR mutant showed the same growth pattern with glucose as the original mutant CHR95.

Uptake of exogenous compatible solutes is preferred over the synthesis, as it is energetically more favorable to the cells [5]. In C. salexigens, the uptake of ectoine, which can be used as a carbon source as well as an osmoprotectant, is maximal at optimal salinity and minimal at low salinity, suggesting that ectoine transport is osmoregulated and most probably devoted to ectoine accumulation from the external medium. In agreement with these transport data, ectoine(s) can be used as carbon source(s) at optimal but not at low salinity [25]. Our previous studies on glucose and ectoine metabolism in this microorganism showed that glucose represses partially ectoine catabolism [25]. However, strain CHR95, which was affected in the transport and metabolism of glucose, did not show an enhanced catabolism of ectoine. These observations indicate that the ability of CHR95 to use ectoine(s) as carbon source at low salinity is decoupled from its impaired glucose catabolism. Rather, it was related to a deregulated ectoine uptake, especially at low salinity. Our results suggest that this phenotype is due to the lack of the two-component response regulator EupR, as a single eupR mutant reproduced the ability of CHR95 to use ectoine(s) as carbon source(s) at low salinity. Preliminary data on the expression of a transcriptional fusion between the $C$. salexigens tea $A$ gene, encoding the ectoine binding protein of the TRAP-transporter for ectoine(s), and the lac $Z$ reporter gene, revealed that expression of teaA in an eupR mutant at $0.75 \mathrm{M} \mathrm{NaCl}$ is $66 \%$ higher than in the wild type (J. Rodriguez-Moya, unpublished results), supporting the hypothesis that 
EupR is involved in the transcriptional control of ectoine uptake.

In the closely related $H$. elongata, the tea $A B C$ genes (encoding the osmoregulated TRAP transporter for ectoine) are followed by teaD, encoding a putative universal stress protein (USP). Deletion of teaD resulted in an enhanced uptake for ectoine by the transporter TeaABC, but it did not affect teaA mRNA-levels, excluding a transcriptional regulation mechanism for TeaD. Rather, TeaD was suggested to function either as a translational regulator or as a direct/indirect regulator of TeaABC transport activity [44]. EupR and TeaD proteins do not show homology to each other, as they belong to different protein families and do not share functional domains. Thus, whereas $H$. elongata TeaD shows the conserved sensory domain of cytoplasmic proteins of the Universal stress protein family [44], C. salexigens EupR contains a single $\mathrm{N}$-terminal receiver domain and a C-terminal HTH DNA-binding domain of the NarL/FixJ family of response regulators [14,17]. As judged by the fact that the eupR mutant is salt-sensitive and grows slower than the wild type with glucose, most probably EupR regulates other processes, besides ectoine uptake, which may or may not be related to the osmostress response. This seems to be also the case of OmpR and MtrA, two response regulators involved in osmoadaptation in E. coli [13] and C. glutamicum [11], respectively. Our phylogenetic analysis grouped EupR with proteins of unknown functions. Its closest characterized relative was the E. coli NarL, which is responsible for the control of nitrate- and nitrite-regulated gene expression [33]. However, assigning protein function based on the function of its closest experimentally characterized homolog is not readily applicable to signal transduction components, as proteins with very similar sequences may have dramatically different biological functions [39]. Therefore, we cannot infer a role of EupR in nitrateand nitrite-regulated gene expression, besides its involvement in the control of ectoine uptake.

The typical scheme of bacterial two-component signal transduction involves signal sensing by a sensory histidine kinase that leads to its autophosphorylation, followed by phosphoryl transfer to Asp residue in the $\mathrm{N}$-terminal REC domain of the cognate response regulator [16]. However, the cognate response regulator and the histidine kinase are not always encoded in close proximity to each other, which complicates their identification [14]. In any case, presence of a gene in the neighborhood of a response regulator could strengthen the case for the analyzed protein being a histidine kinase [39]. The gene Csal869, located three genes downstream of eupR, was predicted to be the cognate histidine kinase associated to EupR. This protein satisfies all the key criteria to be considered as the sensory hybrid histidine kinase. The N-terminal sensor domains of the histidine kinases vary greatly in sequence, membrane topology, composition, and domain arrangement. This variability presumably reflects different principles in stimulus perception and processing. For instance, E. coli $\mathrm{KdpD}$ seems to have a cytoplasmic sensor domain (for $\mathrm{K}^{+}$)and also a transmembrane-associated sensing mechanism (osmolality) [15]. The histidine kinase putatively associated to EupR showed two sensor domains. The first one was predicted to form twelve transmembrane helices and was homologous to sodium/solute symporters (SSSF domain). The stimuli sensed by transmembrane sensory domains such as SSF are membrane associated or occur directly within the membrane interface. They include turgor and mechanical stress, ion or electrochemical gradients and transport processes. For instance, the SSF domain is present in E. coli PutP [45], which uses the free energy stored in electrochemical $\mathrm{Na}^{+}$gradients for the uptake of the compatible solute proline. The second sensory domain was predicted to be cytoplasmatic, and showed two PAS subdomains followed by a C-terminal PAC subdomain. Cytoplasmic sensor domains such as PAS detect the presence of cytoplasmic solutes or respond to diffusible or internal stimuli, such as $\mathrm{O}_{2}$ or $\mathrm{H}_{2}$, or stimuli transmitted by transmembrane sensors.

This redundancy of sensory domains is not rare in nature and in fact a large number of sensor kinases harbor more than one (putative) input domain [15]. The most obvious explanation for the presence of two sensor domains in the protein kinase putatively associated to EupR is that it could sense both external and internal conditions and integrate them. This will be the focus of a further work.

\section{Conclusions}

This work paves the way to the elucidation of the osmosensing and signal transduction pathway leading to the control of ectoine uptake in the model halophilic bacterium C. salexigens. Through the characterization of the salt-sensitive mutant CHR95, we found the gene eupR, encoding a two-component response regulator of the NarL/FixJ family of transcriptional regulators. In our view, the original annotation of EupR as a "two component LuxR family transcriptional regulator" was imprecise, as the EupR protein is not involved in quorum sensing. However, it was precisely annotated in the specialized Signaling Census database, and further confirmed by our phylogenetic analysis, as a response regulator of the NarL/FixJ family. Our results suggest that EupR is not only involved in the control of ectoine uptake, but also in other processes that might or not be related to the C. salexigens osmostress response. Finally, our bioinformatic analysis predicted that the gene 
csal869 encodes a multi sensor hybrid histidine protein kinase which could be the sensory partner of EupR. The presence of two sensor domains in this protein suggest that it could participate in the cross-talk between different signal transduction pathways, as it might be able to sense both external (ions gradient, turgor stress, transport) and internal (cytoplasmatic solutes or proteins, redox state) conditions and integrate them. Future work should focus on (i) elucidating the EupR regulon through transcriptomic analysis, (ii) the in vivo analysis of the role of Csal869 as the cognate protein histidine of EupR, and (iii) investigating if the putative EupR histidine kinase could sense the presence of solutes such as ectoine(s) during uptake.

\section{Methods}

\section{Bacterial strains, plasmids and growth conditions}

The bacterial strains and plasmids used in this study are described in Table 3. Strain CHR61, a spontaneous $\mathrm{Rf}^{\mathrm{r}}$ mutant of C. salexigens DSM 3043, was used as the wild type strain. CHR61 displays wild type growth at all conditions tested. C. salexigens strains were routinely grown in complex SW-2 medium containing $2 \%(\mathrm{w} / \mathrm{v})$ total salts Escherichia coli was grown aerobically in complex Luria-Bertani (LB) medium M63 [48], which contains 20 $\mathrm{mM}$ glucose as the sole carbon source, was used as minimal medium for $C$. salexigens. The osmotic strength of M63 was increased by the addition of a 0.6 to $2.5 \mathrm{M}$ final concentration of $\mathrm{NaCl}$. Although $C$. salexigens can grow in M63 with $0.5 \mathrm{M} \mathrm{NaCl}$, growth is extremely slow at this salinity, and cells take a very long time to reach exponential phase. Therefore, we used M63 with 0.6- $0.75 \mathrm{M} \mathrm{NaCl}$ as the standard medium for a low salt concentration in all experiments. The $\mathrm{pH}$ of all media was adjusted to 7.2 with $\mathrm{KOH}$. Solid media contained $20 \mathrm{~g}$ of Bacto agar per liter (Difco). Otherwise stated, cultures were incubated at $37^{\circ} \mathrm{C}$ in an orbital shaker at $200 \mathrm{rpm}$. When used, filter-sterilized antibiotics were added at the following final concentrations $\left(\mu \mathrm{g} \mathrm{ml}{ }^{-1}\right)$ : ampicillin (Ap), 150 for E. coli; chloramphenicol, 25 for E. coli; gentamicin (Gm), 20 for E. coli and 25 for C. salexigens; kanamycin (Km), 50 for $E$. coli and 75 for C. salexigens; rifampin (Rf), 25 for E. coli and C. salexigens; streptomycin (Sm), 20 for E. coli and 50 for C. salexigens and geneticin (Gn), 20 for for E. coli and $C$. salexigens. When used as the sole carbon sources,

Table 3 Bacterial strains and plasmids used in this study

\begin{tabular}{|c|c|c|}
\hline Strain or plasmid & Relevant genotype and/or description & $\begin{array}{l}\text { Source or } \\
\text { reference }\end{array}$ \\
\hline \multicolumn{3}{|l|}{ C. salexigens strains } \\
\hline DSM $3043^{\top}$ & Wild type & [19] \\
\hline CHR61 & Spontaneous Rf' mutant of C. salexigens DSM 3043 & {$[21]$} \\
\hline CHR95 & 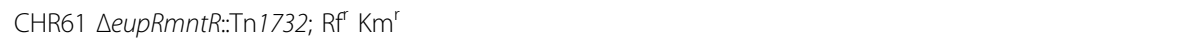 & This study \\
\hline CHR161 & CHR61 mntR:: $2 ; \mathrm{Rf}^{r} \mathrm{Sm}^{\mathrm{r}} \mathrm{Spcr}$ & This study \\
\hline CHR183 & CHR61 eupR:: $\Omega ; \mathrm{Rf}^{r} \mathrm{Gn}^{\mathrm{r}}$ & This study \\
\hline \multicolumn{3}{|l|}{ E. coli strain } \\
\hline $\mathrm{DH} 5 \alpha$ & supE44 $\triangle($ lac) U169 $\varphi 80 \mathrm{~d} / a c Z \Delta M 15$ hsdR17 recA1 endA1 gyrA96 thi-1 relA1; host for DNA manipulations & [65] \\
\hline \multicolumn{3}{|c|}{ 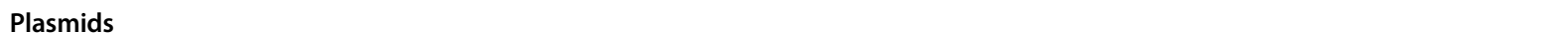 } \\
\hline pKS(-) & Cloning vector; $A p^{r}$ & Stratagene \\
\hline $\mathrm{pHP} 45 \Omega$ & pBR322 derivative carrying the $\Omega$ cassette; $\mathrm{Ap}^{r} \mathrm{Smr} \mathrm{Sp}^{r}$ & [50] \\
\hline 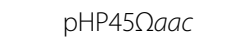 & pBR322 derivative carrying the $\Omega a a c$ cassette; $A p^{r} \mathrm{Gm}^{r} \mathrm{Gn}^{r}$ & [51] \\
\hline pRK600 & Helper plasmid; $\mathrm{Cm}^{r}$ tra & [66] \\
\hline pJQ200-SK & Suicide vector; $G m^{r}$ mob sac & [52] \\
\hline $\begin{array}{l}\text { pSUP102-Gm:: } \\
\text { Tn1732 }\end{array}$ & Mutagenesis plasmid carrying $\mathrm{Tn} 1732 ; \mathrm{Cm}^{\mathrm{r}} \mathrm{Km}^{\mathrm{r}} \mathrm{Gmr}$ & {$[40,49]$} \\
\hline pRR1 & pKS derivative carrying a 20.8-kb sacl fragment from CHR95 including Tn1732; $\mathrm{Ap}^{r} \mathrm{Km}^{\mathrm{r}}$ & This study \\
\hline pMntREupR & $\begin{array}{l}\text { 3-kb Xbal-Apal fragment from C. salexigens genome (containing orf1, eupR, mntR, orf4) cloned into pKS; } \\
\mathrm{Ap}^{r}\end{array}$ & This study \\
\hline pHpalMntr & pMntREupR derivative containing a Hpal recognition site within $m n t R ; A p^{r}$ & This study \\
\hline pHindllIEupR & pMntREupR derivative containing a Hindlll recognition site within eupR; $\mathrm{Ap}^{r}$ & This study \\
\hline$P \Omega M n t R$ & pHpalMntr derivative with $\Omega$ cassette within $m n t R ; \mathrm{Ap}^{r} \mathrm{Sm}^{r} \mathrm{Sp}^{r}$ & This study \\
\hline $\mathrm{p} \Omega$ EupR & pHindlIIEupR derivative with $\Omega a a c$ cassette within eupR; $A p^{r} G^{r} G^{r}$ & This study \\
\hline pJQMntR & $\begin{array}{l}\text { 5-kb Xbal-Apal fragment from } \mathrm{p} \Omega \mathrm{MntR} \text { (containing orf1, eupR, mntR:: } \Omega \text {, orf4) cloned into pJQ200-SK; } \\
\mathrm{Gm}^{r} \mathrm{Sm}^{r} \mathrm{Sp}^{r}\end{array}$ & This study \\
\hline pJQEupR & $\begin{array}{l}\text { 5-kb Xbal-Apal fragment from } \mathrm{p} \Omega \text { EupR (containing orf1, eupR:: } \Omega a a c, m n t R \text {, orf4) cloned into pJQ200-SK; } \\
\mathrm{Gm}^{r} \mathrm{Gn}^{r}\end{array}$ & This study \\
\hline
\end{tabular}


ectoine and hydroxyectoine (bitop AG, Witten, Germany) were added to the media at a final concentration of $20 \mathrm{mM}$. Growth was monitored as the optical density of the culture at $600 \mathrm{~nm}\left(\mathrm{OD}_{600}\right)$ with a Perkin-Elmer Lambda $25 \mathrm{UV} /$ Vis spectrophotometer.

\section{Conjugal transfer of plasmids}

Plasmids were transferred from E. coli to C. salexigens by triparental mating on SW-2 medium, using pRK600 as a helper plasmid, as described by Vargas et al. [46].

\section{Methods for nucleic acid manipulation}

Plasmid DNA was isolated from E. coli with a Wizard Plus SV miniprep kit (Promega), and genomic DNA was isolated with a SpinClean Genomic DNA Purification Kit (Mbiotech). Restriction enzyme digestion and ligation were performed as recommended by the manufacturers (Amersham-Pharmacia Biotech and Fermentas). DNA sequencing was performed by Newbiotechnic (Seville, Spain).

Transposon mutagenesis was performed by conjugal transfer of pSUP102-Gm::Tn1732 from E. coli SM10 $[40,49]$ to C. salexigens strain CHR61. Matings were carried out by mixing the donor and recipient cultures at a ratio of 1:4 (100 $\mu \mathrm{l}$ of donor, $400 \mu \mathrm{l}$ of recipient). The mixed cultures were washed with sterile SW-2 medium to eliminate the antibiotics. The pellet was resuspended in $100 \mu \mathrm{l}$ of SW-2 and placed on a $0.45-\mu \mathrm{m}$ pore filter on SW-2 solid media (which allows the growth of $E$. coli and the putative salt-sensitive mutants of $C$. salexigens). After overnight incubation at $30^{\circ} \mathrm{C}$, cells were resuspended in $20 \%(\mathrm{v} / \mathrm{v})$ sterile glycerol and, after appropriate dilutions, inoculated on SW-2 + rifampicin $+\mathrm{Km}$ plates at a density resulting in about 100-200 colonies per plate. Colonies from these master plates were transferred with sterile toothpicks to duplicate M63 plates, one contained $2.7 \mathrm{M} \mathrm{NaCl}$ and the other contained 0.5 $\mathrm{M} \mathrm{NaCl}$. Plates were incubated at $37^{\circ} \mathrm{C}$ and inspected for colonies that had grown at $0.5 \mathrm{M}$ but not at $2.7 \mathrm{M}$ $\mathrm{NaCl}$. One of these colonies was selected for further experiments and was named CHR95.

To clone the DNA region flanking the Tn1732 insertion in CHR95, genomic DNA of this mutant was digested with SacI, ligated to SacI-digested pKS(-) and the ligation mix was used to transform E. coli DH5 $\alpha$ cells. From $\mathrm{Km}^{\mathrm{r}} \mathrm{Ap}^{\mathrm{r}}$ colonies, the plasmid pRR1, containing the transposon Tn1732 within one SacI fragment of about 20.7-kb, was isolated.

To generate C. salexigens mutants affected in $m n t R$ or eupR, a 3.054-bp fragment from genome containing 680 bp of orf1, eupR, mntR and orf4 was PCR amplified with $P f u$ Turbo DNA polymerase (Stratagene) by using two synthetic oligonucleotides (MntRfw: 5'-CATGCTGATCT AGACGCTGTCGG-3' and MntRrv: 5'-GCAGGCG $\overline{\overline{\text { GGC }}}$
CCCATCTG-3') that were modified (residues in bold) to introduce a $\mathrm{Xba \textrm {I }}$ and an ApaI site, respectively (underlined). The resulting PCR fragment was digested with $X b a \mathrm{I}$ and $A p a \mathrm{I}$, and the 3,054-bp fragment generated was cloned into pKS bluescript to give plasmid pMntREupR. Subsequently, an HpaI or HindIII recognition site was introduced in $m n t R$ or eupR respectively, using the PCRbased QuikChange Site-Directed Mutagenesis Kit (Stratagene) and the following oligonucleotides: MntRHpa_fw: 5' CCGAATTGGTCGAGGACTATGTTAACGAGATTG CGCATTTGC-3', MntRHpa_rv: 5'-GCAAATGCGCA ATCTCGTTAACATAGTCCTCGACCAATTCGG-3', EupRHind_fw: 5'-GCACGGCGCACCACCGGCGAAGC TTCGCTTCCCCAGATGACC-3', and EupRHind_rv: 5'-GGTCATCTCGGGAAGCGAAGCTTCGCCGGTGGT GCGCCGTGC-3', that were modified (residues in bold) to introduce the corresponding restriction sites. The resultant plasmids, pHpaIMntR and pHindIIIEupR were linearized with the enzyme HpaI or HindIII and ligated to 2-kb SmaI or HindIII fragments from pHP45- $\Omega$ [50] or pHP45$\Omega$ aac [51], containing the $\Omega$ interposons for insertional mutagenesis $\left(\mathrm{Sm}^{\mathrm{r}}\right.$ or $\left.\mathrm{Gn}^{\mathrm{r}}\right)$. The resulting plasmids were named $\mathrm{p} \Omega$ MntR and $\mathrm{p} \Omega$ EupR. To recombine the $m n t R$ or eupR mutations into the $C$. salexigens chromosome, $5-\mathrm{kb}$ $X$ baI-ApaII fragments from $\mathrm{p} \Omega M n t R$ or $\mathrm{p} \Omega$ EupR were cloned into the suicide vector PJQSK200 $\left(\mathrm{Gm}^{\mathrm{r}}\right)$ [52] to give plasmids PJQMntR and PJQEupR, which were mobilized into the $C$. salexigens wild type strain by triparental mating. Mutant strains resulting from a double homologous recombination event were identified as $\mathrm{Sm}^{\mathrm{r}} \mathrm{Gm}^{\mathrm{s}}$, or $\mathrm{Gn}^{\mathrm{r}} \mathrm{Gm}^{\mathrm{s}}$ colonies on SW-2 plates containing $10 \%$ sucrose. Two of these colonies were purified for further analysis and were named CHR161 (mntR:: $\Omega$ ) and CHR183 (eupR:: $\Omega a a c)$. Insertions of the omega cassette in CHR161 and CHR183 were confirmed by PCR and sequencing.

\section{Determination of sensitivity to $\mathrm{Mn}$}

To determine the sensitivity of $C$. salexigens strains to $\mathrm{Mn}$, we used fresh plates of a modified SW-2 medium containing less than $1 \mathrm{mM}$ of $\mathrm{SO}_{4} \mathrm{Mg}$ (to avoid interference of $\mathrm{Mg}^{2+}$ with $\mathrm{Mn}^{2+}$ ), which was additioned with 0.5 to $2.5 \mathrm{mM} \mathrm{MnCl} 2$. An overnight culture of each strain $(100 \mu \mathrm{l})$ was spread onto the assay plate and growth was observed after incubation at $37^{\circ} \mathrm{C}$ for $48 \mathrm{~h}$.

\section{Determination of ectoine uptake}

Cells grown overnight in SW-2 were subcultured at a 1:100 dilution in glucose M63 medium containing 0.75, 1.5 or $2.5 \mathrm{M}$ of $\mathrm{NaCl}$, and grown up to exponential phase $\left(\mathrm{OD}_{600}\right.$ ca. 0.5$)$. Transport was initiated by adding $\left[{ }^{14} \mathrm{C}\right]$-ectoine to $0.2 \mathrm{ml}$ of bacterial suspensions and incubating the cultures at room temperature. The $\left[{ }^{14} \mathrm{C}\right]$ ectoine (5.5 MBq $\mathrm{mM}$ ) was prepared biologically from Brevibacterium linens as described [53] and was added 
at a final concentration of $87 \mu \mathrm{M}$. During $2 \mathrm{~min}, 50 \mu \mathrm{l}$ of samples were taken at 30-s intervals, and transport was terminated by rapid filtration through Whatman GF/F discs (Fisher Bioblock, Illkirch, France). The cells were quickly washed twice with $2 \mathrm{ml}$ of isotonic M63 medium. The filters were solubilized in scintillation fluid and radioactivity was determined in a Packard Liquid Scintillation Analyzer, 1600 TR (Perkin Elmer, Courtabeouf, France). Transport rates were expressed as nmol min-1 OD-1 unit.

\section{Determination of the metabolic fate of $\left[{ }^{14} \mathrm{C}\right]$-glucose}

Cells grown overnight in SW-2 were subcultured at a 1:100 dilution in $\mathrm{M} 63$ containing $1.5 \mathrm{M} \mathrm{NaCl}$ and 20 $\mathrm{mM}$ glucose, and grown up to exponential phase ${\left(\mathrm{OD}_{600}\right.}$ ca. 0.5). $2 \mathrm{ml}$ samples were centrifuged, resuspended in $1.5 \mathrm{M} \mathrm{NaCl} \mathrm{M} 63$ to an $\mathrm{OD}_{600}$ of $c a .0 .6$ and transferred to a Warburg flask. ${ }^{14} \mathrm{C}$-labelled glucose $(5.5 \mathrm{mCi} / \mathrm{mmol}$, $390000 \mathrm{dpm} / 5 \mu \mathrm{l}$ ) was added at a final concentration of $100 \mu \mathrm{M}$ to the samples. After different incubation times at $37^{\circ} \mathrm{C}, 1 \mathrm{ml}$ of sample was centrifuged for $10 \mathrm{~min}$ at $16000 \mathrm{~g} ; 50 \mu \mathrm{l}$ of supernatant was taken (twice) and radioactivity was measured as above, indicating the glucose remaining in the supernatant $\left(\mathrm{S}, \mathrm{dpm} \mathrm{ml-}{ }^{1}\right)$. Cell pellet was resuspended in $20 \mu \mathrm{l}$ of $\mathrm{H}_{2} \mathrm{O}$, extracted with $80 \mu \mathrm{l}$ of pure ethanol and centrifuged for $10 \mathrm{~min}$ at 13000 rev min ${ }^{-1}$. The ethanolic supernatant was dried in a Speed Vac (Savant Instruments, Holbrook, NY, USA), and the solid residue was resuspended in $50 \mu \mathrm{l}$ of $\mathrm{H}_{2} \mathrm{O}$. An aliquot of $10 \mu \mathrm{l}$ was used to measure the radioactivity caused by the ethanol-soluble 28 compounds synthesized from glucose (ESF, dpm per OD unit). The ethanol insoluble pellet was resuspended in $50 \mu \mathrm{l}$ of $\mathrm{H}_{2} \mathrm{O}$ and used to measure the radioactivity caused by the ethanol-insoluble compounds synthesized from glucose (EIF, dpm per OD unit).

\section{Determination of the metabolic fate of $\left[{ }^{14} \mathrm{C}\right]$-ectoine}

Cells grown overnight in SW-2 were subcultured at a 1:100 dilution in $\mathrm{M} 63$ containing $1.5 \mathrm{M} \mathrm{NaCl}$ and 20 $\mathrm{mM}$ glucose and grown up to exponential phase $\left(\mathrm{OD}_{600}\right.$ $c a$. 0.5). Two independent $2 \mathrm{ml}$ samples were centrifuged, resuspended in $1.5 \mathrm{M} \mathrm{NaCl} \mathrm{M63} \mathrm{to} \mathrm{an} \mathrm{OD}_{600}$ of ca. 0.6 and transferred to a Warburg flask. ${ }^{14} \mathrm{C}$-labelled ectoine (5.5 MBq $\mathrm{mM}$ ) was added at a final concentration of $87 \mu \mathrm{M}$ to the samples. Glucose was added to one of the samples at a final concentration of $20 \mathrm{mM}$. After 2 -h incubation at $37^{\circ} \mathrm{C}$, the fate of radioactive ectoine was analysed as follows: (i) respired radioactive $\mathrm{CO}_{2}$ was trapped on a strip of $3 \mathrm{MM}$ Whatman filter paper moistened with $50 \mu \mathrm{l}$ of $6 \mathrm{~mol} \mathrm{l-}^{1}$ of $\mathrm{KOH}$ and ${ }^{14} \mathrm{CO}_{2}$ production (dpm per $\mathrm{OD}_{600}$ unit) was measured by liquid scintillation; (ii) $1 \mathrm{ml}$ of sample was centrifuged for $10 \mathrm{~min}$ at $16000 \mathrm{~g}$; $50 \mu \mathrm{l}$ of supernatant was taken (twice) and radioactivity was measured as above, indicating the ectoine remaining in the supernatant $(\mathrm{S}$, $\mathrm{dpm} \mathrm{ml-}{ }^{1}$ ); and (iii) cell pellet was resuspended in $20 \mu \mathrm{l}$ of $\mathrm{H}_{2} \mathrm{O}$, extracted with $80 \mu \mathrm{l}$ of pure ethanol and centrifuged for $10 \mathrm{~min}$ at $13000 \mathrm{rev} \mathrm{min}^{-1}$. The ethanolic supernatant was dried in a Speed Vac (Savant Instruments, Holbrook, NY, USA), and the solid residue was resuspended in $50 \mu \mathrm{l}$ of $\mathrm{H}_{2} \mathrm{O}$. An aliquot of $10 \mu \mathrm{l}$ was used to measure the radioactivity caused by the ethanolsoluble compounds synthesized from ectoine (ESF, dpm per OD unit). The ethanol insoluble pellet was resuspended in $50 \mu \mathrm{l}$ of $\mathrm{H}_{2} \mathrm{O}$ and used to measure the radioactivity caused by the ethanol-insoluble compounds synthesized from ectoine (EIF, dpm per OD unit).

\section{Phylogenetic analysis}

Phylogenetic and molecular evolutionary analyses were conducted using MEGA version 4 [54]. C. salexigens EupR and other LuxR family proteins including well characterized members of different subclasses with a common LuxR-C-like conserved domain and others different domains were included in the phylogenetic analyses. We also included some uncharacterized proteins with a high similarity to $C$. salexigens EupR, including two paralogs present in C. salexigens genome.

The sequences were aligned with clustalW (1.6) using a BLOSUM62 matrix and manually edited. The phylogenetic tree was inferred using the Neighbor-joining method [55] and the evolutionary distances were computed using the Poisson correction method. The rate variation among sites was modelled with a gamma distribution (shape parameter $=1.5$ ) and all the positions containing gaps and missing data were eliminated only in pairwise sequence comparisons. The robustness of the tree branches was assessed by performing bootstrap analysis of the Neighbor-joining data based on 1000 resamplings [56].

\section{DNA and protein sequences analysis}

The sequence of the $C$. salexigens genome is available at NCBI microbial genome database (http://www.ncbi.nlm. nih.gov/genomes/lproks.cgi Ac $\mathrm{N}^{\circ}$ : NC_007963). Sequence data were analyzed using PSI-BLAST at NCBI server http://www.ncbi.nlm.nih.gov/BLAST. Promoter sequences were predicted using BGDP Neural Network Promoter Prediction http://www.fruitfly.org/seq_tools/promoter. $\mathrm{html}$. Signal peptides and topology of proteins were predicted using SMART 6 (http://smart.embl-heidelberg.de/; $[57,58])$. Other programs and databases used in proteins topology and functional analysis were STRING 8.2 (http:// string.embl.de/; [38]) KEGG (http://www.genome.ad.jp/ kegg/pathway/ko/ko02020.html; [59]), Signaling census (http://www.ncbi.nlm.nih.gov/Complete_Genomes/SignalCensus.html; [28,29]), PROSITE (http://www.expasy.org/ 
prosite/; [60]), BLOCKS (http://blocks.fhcrc.org/; [61]), Pfam (http://pfam.janelia.org/; [62]), CDD (http://www. ncbi.nlm.nih.gov/Structure/cdd/cdd.shtml; [27]), InterProScan (http://www.ebi.ac.uk/interpro/; [63]), and Phobius (http://www.ebi.ac.uk/Tools/phobius/; [64]).

\section{Acknowledgements}

This research was financially supported by grants from the Spanish Ministerio de Ciencia e Innovación (BIO2008-04117), and Junta de Andalucía (P08-CVI-03724). Javier Rodriguez-Moya and Mercedes Reina-Bueno were recipients of a fellowship from the Spanish Ministerio de Educación y Ciencia.

\section{Author details}

'Department of Microbiology and Parasitology, Faculty of Pharmacy, University of Seville, Seville, Spain. ${ }^{2}$ Department of Organic and Pharmaceutical Chemistry. Faculty of Pharmacy, University of Seville, Seville, Spain. ${ }^{3}$ Laboratoire Interactions Cellulaires et Moléculaires, DUALS, CNRS 6026, Université de Rennes I, Campus Beaulieu, 35042 Rennes Cedex, France. ${ }^{4}$ Laboratoire de Microbiologie des Environnements Extrêmes - LM2E (CNRS), Université de Bretagne Occidentale, Plouzané, France.

\section{Authors' contributions}

JRM and MA performed the majority of the experiments, participated in bioinformatics analysis, study design, and in crafting of the manuscript. MRB, MJ, and FIG performed some growth experiments and RMN analyses. JJN and CV conceived the study, participated in the design, coordination, bioinformatic analysis, and crafting of the manuscript. All authors have read and approved the final manuscript.

\section{Received: 23 March 2010 Accepted: 13 October 2010}

Published: 13 October 2010

\section{References}

1. Bremer E, Krämer R: Coping with osmotic challenges: osmoregulation trough accumulation and release of compatible solutes in bacteria. In Bacterial Stress Responses. Edited by: Storz G, Hengge-Aronis R. Washington DC: ASM Press; 2000:79-97.

2. Wood JM, Bremer E, Csonka LN, Krämer R, Poolman B, van der Heide $T$, Smith LT: Osmosensing and osmoregulatory compatible solutes accumulation by bacteria. Comp Biochem Physiol 2001, 130:437-460.

3. Galinski EA, Trüper HG: Microbial behaviour in salt-stressed ecosystems. FEMS Microbiol Rev 1994, 15:95-108.

4. Welsh DT: Ecological significance of compatible solute accumulation by micro-organisms: from single cells to global climate. FEMS Microbiol Rev 2000, 24:263-290.

5. Oren A: Bioenergetic aspects of halophilism. Microbiol Mol Biol Rev 1999, 63:334-348.

6. Booth IR, Edwards MD, Black S, Schumann U, Miller S: Mechanosensitive channels in bacteria: signs of closure? Nat Rev Microbiol 2007, 6:431-440.

7. Jebbar M, Sohn-Bösser L, Bremer E, Bernard T, Blanco C: Ectoine-induced proteins in Sinorhizobium meliloti include an Ectoine ABC-type transporter involved in osmoprotection and ectoine catabolism. $J$ Bacteriol 2005, 187:1293-1304

8. Vargas C, Argandoña M, Reina-Bueno M, Rodríguez-Moya J, FernándezAunión C, Nieto Jj: Unravelling the adaptation responses to osmotic and temperature stress in Chromohalobacter salexigens, a bacterium with broad salinity tolerance. Saline Systems 2008, 4:14.

9. Wood JM: Bacterial osmosensing transporters. Methods Enzymol 2007, 428:77-107.

10. Grammann K, Volke A, Kunte HJ: New type of osmoregulated solute transporter identified in halophilic members of the bacteria domain: TRAP transporter TeaABC mediates uptake of ectoine and hydroxyectoine in Halomonas elongata DSM 2581(T). J Bacteriol 2002, 184:3078-3085

11. Krämer R: Osmosensing and osmosignaling in Corynebacterium glutamicum. Amino Acids 2009, 37:487-497.
12. Hamann K, Zimmann $P$, Altendorf K: Reduction of turgor is not the stimulus for the sensor kinase KdpD of Escherichia coli. J Bacteriol 2008, 190:2360-2367.

13. Jung K, Hamann $K$, Revermann A: K+ stimulates specifically the autokinase activity of purified and reconstituted EnvZ of Escherichia coli. J Biol Chem 2001, 276:40896-40902.

14. Gao R, Mack TR, Stock AM: Bacterial response regulators: versatile regulatory strategies from common domains. Trends Biochem Sci 2007, 32:225-234.

15. Mascher T, Helmann JD, Unden G: Stimulus perception in bacterial signaltransducing histidine kinases. Microbiol Mol Biol Rev 2006, 70:910-938.

16. Stock AM, Robinson VL, Goudreau PN: Two-component signal transduction. Annu Rev Biochem 2000, 69:183-215.

17. Galperin MY: Structural classification of bacterial response regulators: Diversity of output domains and domains combinations. J Bacterio/ 2006, 188:4169-4182.

18. Koretke KK, Lupas AN, Warren PV, Rosenberg M, Brown JR: Evolution of two-component signal transduction. Mol Biol Evol 2000, 17:1956-1970.

19. Arahal DR, García MT, Vargas C, Cánovas D, Nieto JJ, Ventosa A: Chromohalobacter salexigens sp. nov., a moderately halophilic species that includes Halomonas elongata DSM 3043 and ATCC 33174. Int J System Evol Microbiol 2001, 51:1457-1462.

20. Canovas D, Vargas C, Csonka LN, Ventosa A, Nieto JJ: Osmoprotectants in Halomonas elongata: high-affinity betaine transport system and cholinebetaine pathway. J Bacteriol 1996, 178:7221-7226.

21. Cánovas D, Vargas C, Iglesias-Guerra F, Csonka LN, Rhodes D, Ventosa A, Nieto JJ: Isolation and characterization of salt-sensitive mutants of the moderate halophile Halomonas elongata and cloning of the ectoine synthesis genes. J Biol Chem 1997, 272:25794-25801.

22. García-Estepa R, Argandoña M, Reina-Bueno M, Capote N, Iglesias-Guerra F, Nieto JJ, Vargas C: The ectD gene, which is involved in the synthesis of the compatible solute hydroxyectoine, is essential for thermo protection of the halophilic bacterium Chromohalobacter salexigens. J Bacteriol 2006, 188:3774-3784

23. Cánovas D, Vargas C, Calderon MI, Ventosa A, Nieto JJ: Characterization of the genes for the biosynthesis of the compatible solute ectoine in the moderately halophilic bacterium Halomonas elongata DSM3043. System Appl Microbiol 1998, 21:487-497.

24. Calderón MI, Vargas C, Rojo F, Iglesias-Guerra F, Csonka LN, Ventosa A, Nieto JJ: Complex regulation of the synthesis of the compatible solute ectoine in the halophilic bacterium Chromohalobacter salexigens DSM3043T. Microbiology 2004, 150:3051-3063.

25. Vargas C, Jebbar M, Carrasco R, Blanco C, Calderón Ml, Iglesias-Guerra F, Nieto JJ: Ectoines as compatible solutes and carbon and energy sources for the halophilic bacterium Chromohalobacter salexigens. J Appl Microbiol 2006, 100:98-107.

26. Moore C, Helmann JD: Metal ion homeostasis in Bacillus subtilis. Curr Opin Microbiol 2005, 8:188-195

27. Marchler-Bauer A, Bryant SH: CD-Search: protein domain annotations on the fly. Nucleic Acids Res 2004, 32:W327-331.

28. Galperin MY: A census of membrane-bound and intracellular signal transduction proteins in bacteria: bacterial IQ, extroverts and introverts. BMC Microbiol 2005, 14(5):35

29. Galperin MY, Higdon R, Kolker E: Interplay of heritage and habitat in the distribution of bacterial signal transduction systems. Mol BioSyst 2010, 6:721-728.

30. Aravind L, Anantharaman V, Balaji S, Babu MM, lyer LM: The many faces of the helix-turn-helix domain: transcription regulation and beyond. FEMS Microbiol Rev 2005, 29:231-262.

31. Foussard M, Garnerone AM, Ni F, Soupène E, Boistard P, Batut J: Negative autoregulation of the Rhizobium meliloti fixK gene is indirect and requires a newly identified regulator, FixT. Mol Microbiol 1997, 25 27-37.

32. Olekhnovich IN, Kadner RJ: Mutational scanning and affinity cleavage analysis of UhpA-binding sites in the Escherichia coli uhpT promoter. $J$ Bacteriol 2002, 184:2682-2691

33. Rabin RS, Stewart V: Dual response regulators (NarL and NarP) interact with dual sensors (NarX and NarQ) to control nitrate- and nitriteregulated gene expression in Escherichia coli K-12. J Bacteriol 1993, 175:3259-3268. 
34. Gambello MJ, Iglewski BH: Cloning and characterization of the Pseudomonas aeruginosa las $R$ gene, a transcriptional activator of elastase expression. J Bacteriol 1991, 173:3000-3009.

35. McGowan S, Sebaihia M, Jones S, Yu B, Bainton N, Chan PF, Bycroft B, Stewart GS, Williams P, Salmond GP: Carbapenem antibiotic production in Erwinia carotovora is regulated by CarR, a homologue of the LuxR transcriptional activator. Microbiology 1995, 141:541-550.

36. Ducros VM, Lewis RJ, Verma CS, Dodson EJ, Leonard G, Turkenburg JP, Murshudov GN, Wilkinson AJ, Brannigan JA: Crystal structure of GerE, the ultimate transcriptional regulator of spore formation in Bacillus subtilis. J Mol Biol 2001, 306:759-771.

37. Dubern JF, Coppoolse ER, Stiekema WJ, Bloemberg GV: Genetic and functional characterization of the gene cluster directing the biosynthesis of putisolvin I and II in Pseudomonas putida strain PCL1445. Microbiology 2008, 154:2070-2083

38. Jensen $\sqcup$, Kuhn M, Stark M, Chaffron S, Creevey C, Muller J, Doerks T, Julien P, Roth A, Simonovic M, Bork P, von Mering C: STRING 8-a global view on proteins and their functional interactions in 630 organisms. Nucleic Acids Res 2009, 37:D412-D416.

39. Galperin MY, Nikolskaya AN: Identification of sensory and signaltransducing domains in two-component signalling systems. Methods Enzymol 2007, 422:47-74.

40. Ubben D, Schmitt R: Tn1721 derivatives for transposon mutagenesis, restriction mapping and nucleotide sequence analysis. Gene 1986, 41:145-152.

41. Vargas C, Nieto JJ: Genetic tools for the manipulation of moderately halophilic bacteria of the family Halomonadaceae. In Methods in Molecular Biology. Edited by: Balbás P, Lorence A. Totowa, NJ: Humana Press Inc; 2004:267:183-208.

42. Altenbuchner J, Schmitt R: Transposon Tn1721: site-specific recombination generates deletions and inversions. Mol Gen Genet 1983, 190:300-308

43. Starai VJ, Escalante-Semerena JC: Acetyl-coenzyme A synthetase (AMP forming). Cell Mol Life Sci 2004, 61:2020-2030.

44. Schweikhard ES, Kuhlmann SI, Kunte HJ, Grammann K, Ziegler CM: Structure and function of the universal stress protein TeaD and its role in regulating the ectoine transporter TeaABC of Halomonas elongata DSM 2581T. Biochemistry 2010, 49:2194-2204.

45. Jung $H$ : The sodium/substrate symporter family: structural and functional features. FEBS Lett 2002, 529:73-77.

46. Vargas C, Coronado MJ, Ventosa A, Nieto JJ: Host range, stability, and compatibility of broad host-range-plasmids and a shuttle vector in moderately halophilic bacteria. Evidence of intragenic and intergenic conjugation in moderate halophiles. System Appl Microbiol 1997 20:173-181.

47. Miller JH: A Short Course in Bacterial Genetics. Cold Spring Harbor, NY: Cold Spring Harbor Laboratory 1992.

48. Csonka LN: A third L-proline permease in Salmonella typhimurium which functions in media of elevated osmotic strength. J Bacteriol 1982, 151:1433-1443.

49. Simon $R$, Priefer U, Pühler A: A broad host range mobilization system for in vivo genetic engineering: transposon mutagenesis in Gram-negative bacteria. Biotechnology 1983, 1:784-791.

50. Prentki $P$, Krisch HM: In vitro insertional mutagenesis with a selectable DNA fragment. Gene 1984, 29:303-313.

51. Blondelet-Rouault MH, Weiser J, Lebrihi A, Branny P, Pernodet JL: Antibiotic resistance gene cassettes derived from the $\pi$ interposon for use in $E$. coli and Streptomyces. Gene 1997, 190:315-317.

52. Quandt J, Hynes MF: Versatile suicide vectors which allow direct selection for gene replacement in gram-negative bacteria. Gene 1993, 127:15-21.

53. Jebbar M, Talibart R, Gloux K, Bernard T, Blanco C: Osmoprotection of Escherichia coli by ectoine: uptake and accumulation characteristics. $J$ Bacteriol 1992, 174:5027-5035.

54. Tamura K, Dudley J, Nei M, Kumar S: MEGA4: Molecular Evolutionary Genetics Analysis (MEGA) software version 4.0. Mol Biol Evol 2007, 24:1596-1599.

55. Saitou N, Nei M: The Neighbor-joining method: a new method for reconstructing phylogenetic trees. Mol Biol Evol 1987, 4:406-425.

56. Felsenstein J: Confidence limits on phylogenies: an approach using the bootstrap. Evolution 1985, 39:783-791.
57. Schultz J, Milpetz F, Bork P, Ponting CP: SMART, a simple modular architecture research tool: Identification of signaling domains. Proc Natl Acad Sci USA 1998, 95:5857-5864.

58. Letunic I, Doerks T, Bork P: SMART 6: recent updates and new developments. Nucleic Acids Res 2009, 37:D229-D232.

59. Kanehisa M, Goto S, Kawashima S, Okuno Y, Hattori M: The KEGG resource for deciphering the genome. Nucleic Acids Res 2004, 32:D277-280.

60. Hulo N, Bairoch A, Bulliard V, Cerutti L, Cuche B, De Castro E, Lachaize C, Langendijk-Genevaux PS, Sigrist CJA: The 20 years of PROSITE. Nucleic Acids Res 2008, 36:D245-D249.

61. Henikoff JG, Pietrokovski S, McCallum CM, Henikoff S: Blocks-based methods for detecting protein homology. Electrophoresis 2000 21:1700-1706.

62. Finn RD, Mistry J, Tate J, Coggill P, Heger A, Pollington JE, Gavin OL, Gunesekaran P, Ceric G, Forslund K, Holm L, Sonnhammer EL, Eddy SR, Bateman A: The Pfam protein families database. Nucleic Acids Res 2010, 38:D211-222.

63. Zdobnov EM, Apweiler R: InterProScan - an integration platform for the signature-recognition methods in InterPro. Bioinformatics 2001, 17:847-848.

64. Käll L, Krogh A, Sonnhammer ELL: A Combined Transmembrane Topology and Signal Peptide Prediction Method. J Mol Biol 2004, 338:1027-1036.

65. Hanahan D: Studies on transformation of Escherichia coli with plasmids. $J$ Mol Biol 1983, 166:557-580

66. Kessler B, de Lorenzo V, Timmis KN: A general system to integrate lacZ fusion into the chromosome of gram negative bacteria: regulation of the Pm promoter of the TOL plasmid studied with all controlling elements in monocopy. Mol Gen Genet 1992, 233:293-301.

doi:10.1186/1471-2180-10-256

Cite this article as: Rodríguez-Moya et al.: Involvement of EupR, a response regulator of the NarL/FixJ family, in the control of the uptake of the compatible solutes ectoines by the halophilic bacterium Chromohalobacter salexigens. BMC Microbiology 2010 10:256.

\section{Submit your next manuscript to BioMed Central and take full advantage of:}

- Convenient online submission

- Thorough peer review

- No space constraints or color figure charges

- Immediate publication on acceptance

- Inclusion in PubMed, CAS, Scopus and Google Scholar

- Research which is freely available for redistribution

Submit your manuscript at www.biomedcentral.com/submit
C Biomed Central 